\title{
Resonance and Singularities
}

\author{
Henk W. Broer and Gert Vegter* \\ To Jan Karel Lenstra, on the occasion of his retirement.
}

August 18, 2011

\begin{abstract}
The phenomenon of resonance will be dealt with from the viewpoint of dynamical systems depending on parameters and their bifurcations. Resonance phenomena are associated to open subsets in the parameter space, while their complement corresponds to quasi-periodicity and chaos. The latter phenomena occur for parameter values in fractal sets of positive measure. We describe a universal phenomenon that plays an important role in modelling. This paper gives a summary of the background theory, veined by examples.
\end{abstract}

\section{What is resonance?}

A heuristic definition of resonance considers a dynamical system, usually depending on parameters, with several oscillatory subsystems having a rational ratio of frequencies and a resulting combined and compatible motion that may be amplified as well. Often the latter motion is also periodic, but it can be more complicated as will be shown below. We shall take a rather eclectic point of view, discussing several examples first. Later we shall turn to a number of universal cases, these are context-free models that occur generically in any system of sufficiently high-dimensionial state and parameter space.

Among the examples are the famous problem of Huygens's synchronizing clocks and that of the Botafumeiro in the Cathedral of Santiago de Compostela, but also

${ }^{*}$ Johann Bernoulli Institute for Mathematics and Computer Science, University of Groningen, PO Box 407, 9700 AK Groningen, The Netherlands 

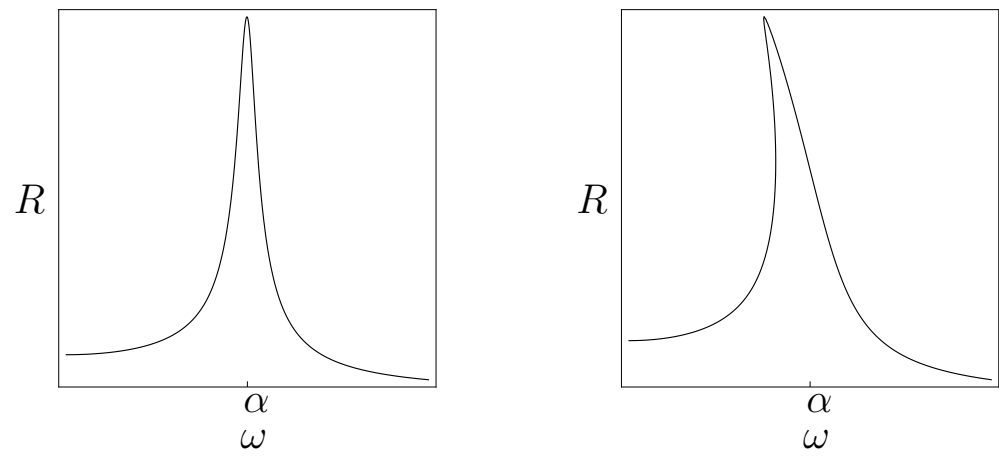

Figure 1: Amplitude-response diagrams in the $(\omega, R)$-plane. Left: the periodically driven harmonic oscillator (1) and right: the driven Duffing oscillator (2).

we briefly touch on tidal resonances in the planetary system. As universal models we shall deal with the Hopf-Nermark-Sacker bifurcation and the Hopf saddlenode bifurcation for mappings. The latter two examples form 'next cases' in the development of generic bifurcation theory. The term universal refers to the context independence of their occurrence: in any with certain generic specifications these bifurcations occur in a persistent way. We witness an increase in complexity in the sense that in the parameter space the resonant phenomena correspond to an open $\&$ dense subset union of tongues, while in the complement of this a nowhere dense set of positive measure exists, corresponding to multi- or quasiperiodic dynamics. This nowhere dense set has a fractal geometry in a sense that will be explained later. From the above it follows that this global array of resonance tongues and fractal geometry has a universal character. As we shall see, both locally and globally Singularity Theory can give organizing principles. It should be noted at once that next to periodic and quasi-periodic dynamics also forms of chaotic dynamics will show up.

Remark. In many cases the resonant bifurcations are repeated at ever smaller scales inside the tongues, leading to an infinite regress. Then we have to extend the notion of open \& dense to residual and that of nowhere dense to meagre. Here a residual set contains a countable intersection of open $\&$ dense sets, while a meagre set is a countable union of nowhere dense sets. One sometimes also speaks in terms of $G_{\delta}-$ or $F_{\sigma}$-sets, respectively [72]. 


\subsection{Periodically driven oscillators}

Many of the resonant phenomena of interest to us are modelled by periodically driven or coupled oscillators. To fix thoughts we now present two examples, namely the harmonic and the Duffing oscillator subjected to periodic forcing.

\subsubsection{The driven harmonic oscillator}

One of the simplest class-room examples of resonance occurs in the harmonic oscillator with periodic forcing

$$
\ddot{x}=-\alpha^{2} x-c \dot{x}+\varepsilon \sin (\omega t)
$$

with $x, t \in \mathbb{R}$, where $c>0$ is the damping and where $\varepsilon \geq 0$ controls the size of the forcing. The forcing has frequency $\omega>0$ and therefore has period $T=2 \pi / \omega$. The 'response' solution is periodic

$$
x(t)=R \sin (\omega t+\phi)
$$

with this same frequency. Its amplitude and phase are given by

$$
R=\frac{\varepsilon}{\sqrt{\left(\omega^{2}-\alpha^{2}\right)^{2}+c^{2} \omega^{2}}} \text { and } \tan \phi=\frac{c \omega}{\omega^{2}-\alpha^{2}} .
$$

Fixing $\alpha, c$ and $\varepsilon$ we consider $R$ as a function of $\omega$, see the amplitude-response diagram in Figure 1 (left) where the response amplitude $R$ is plotted as a function of $\omega$.

\subsubsection{The driven Duffing oscillator}

As a non-linear variation on the above we consider the Duffing equation with periodic forcing

$$
\ddot{x}=-\alpha^{2} x-c \dot{x}-\delta x^{3}+\varepsilon \sin (\omega t),
$$

where $\alpha, \omega, c, \varepsilon$ and are positive and where $\delta$ with $0<\delta \ll 1$ is considered as a perturbation parameter. Fixing $\alpha, c$ and $\varepsilon$ as before, by successive approximation of a solution

$$
x(t)=R \sin (\omega t+\phi),
$$

we obtain the following relation between $\varepsilon$ and $R$ :

$$
\varepsilon^{2}=\left(\left(\omega^{2}-\alpha^{2}\right)^{2}+c^{2} \omega^{2}\right) R^{2}+\frac{3}{2} \delta\left(\omega^{2}-\alpha^{2}\right) R^{4}+\frac{9}{16} \delta^{2} R^{6}+O\left(\delta^{3}\right),
$$


as $\delta \downarrow 0$, with a similar approximation for the phase $\phi$, compare with Stoker [86], also see [24]. In Figure 1 (right) we depict the corresponding curve in the $(\omega, R)-$ plane, which now no longer is a graph.

\section{Remarks}

- One of the exciting things about resonance concerns the peaks of the amplitude $R$ that can be quite high, even where $\varepsilon$ is still moderate. Systems like (1) and (2) form models or metaphors for various resonance phenomena in daily life. In many cases high resonance peaks one needs to 'detune' away from the resonance value corresponding to the peak, think of a marching platoon of soldiers that have to go out of pace when crossing a bridge.

- In other cases, like when 'tuning' the radio receiver to a certain channel, one takes advantage of the peak.

- It should be noted that the nonlinear (2) dynamically is far richer than the linear case (1), e.g., see [57] and references therein.

\subsubsection{Geometrical considerations}

In both cases of the driven oscillators witnessed above the state space is $\mathbb{R}^{2} \times \mathbb{T}^{1}=$ $\{x, y, z\}$, where

$$
\begin{aligned}
\dot{x} & =y \\
\dot{y} & =a(x, y, \delta)+\varepsilon \sin z \\
\dot{z} & =\omega,
\end{aligned}
$$

with $a(x, y, \delta)=-\alpha^{2} x-c y-\delta x^{3}$, where $\delta=0$ in the harmonic example. The second factor is the circle $\mathbb{T}^{1}=\mathbb{R} /(2 \pi \mathbb{Z})$ which takes into account the periodicity of the systems in $z$. The response motion of the form $x(t)=R \sin (\omega t+\phi)$ then corresponds to a closed curve

$$
\left(\begin{array}{l}
x \\
y \\
z
\end{array}\right)(t)=\left(\begin{array}{c}
R \sin (\omega t+\phi) \\
\omega R \cos (\omega t+\phi) \\
\omega t+\phi
\end{array}\right) .
$$

This closed curve, when projected onto the $(x, y)$-plane, exactly forms an ellipse. 


\section{lyupgens' V.') clocks 1665 .

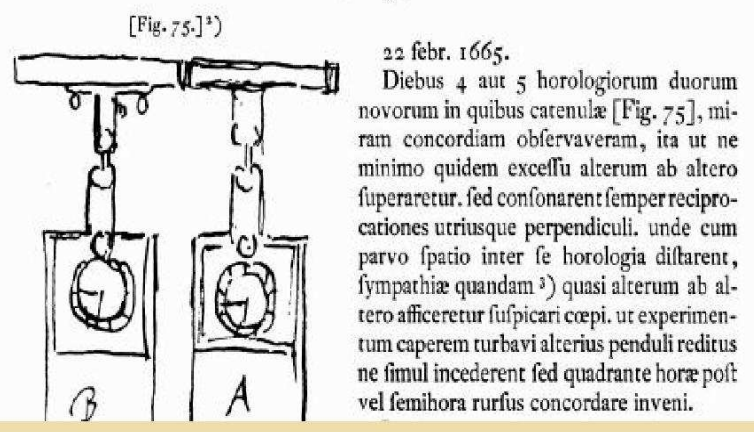

Figure 2: Huygens's synchronizing clocks [60].

We now can describe this motion in terms of a 2-dimensional torus $\mathbb{T}^{2}=\mathbb{T}^{1} \times \mathbb{T}^{1}$, parametrized by two variables $\varphi_{1}$ and $\varphi_{2}$ in a system of differential equations of the format

$$
\begin{aligned}
& \dot{\varphi}_{1}=\omega_{1} \\
& \dot{\varphi}_{2}=\omega_{2},
\end{aligned}
$$

where $\varphi_{1}=z$ and $\omega_{1}=\omega$ and where for $\varphi_{2}$ we take the phase of the motion on the ellipse. Thus $\varphi_{2}$ exactly is the time parametrization of this motion, scaled to the period $2 \pi$ : in this case $\omega_{2}=\omega_{1}=\omega$. Therefore in this way, the curve (3) can be seen as a $1: 1$ torus knot.

To view resonant motion in terms of torus dynamics turns out to be extremely useful and this can also be applied to coupled oscillators. Here a classical example is given by Christiaan Huygens [60], who in 1665 observed the following phenomenon, see Figure 2. Two nearly identical pendulum clocks mounted on a not completely rigid horizontal beam tend to synchronize. Moreover, when the pendula both move in the vertical plane through the beam, they have a tendency to synchronize in anti-phase motion. A simple model describes this system in the format (4), where the angles $\varphi_{1}$ and $\varphi_{2}$ are the phases of the two oscillators and where again $\omega_{1}=\omega_{2}$. Later on we will come back to this and other examples where we will also see other frequency ratios $\omega_{1}: \omega_{2}$. 


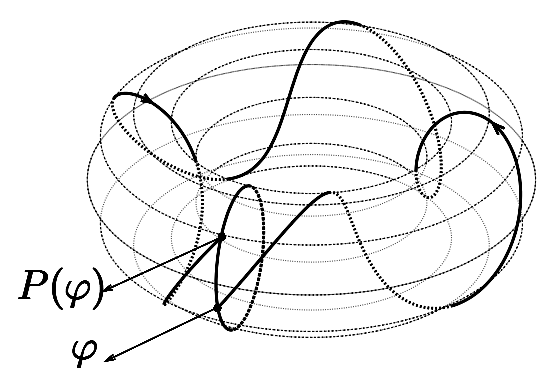

Figure 3: Poincaré mapping of a torus flow [42].

\subsection{Torus flows and circle mappings}

In this section we turn to the dynamics on the 2-dimensional torus $\mathbb{T}^{2}$ from $\S 1.1 .3$ for its own sake, introducing the weakly coupled system

$$
\begin{aligned}
& \dot{\varphi}_{1}=\omega_{1}+\varepsilon f_{1}\left(\varphi_{1}, \varphi_{2}\right) \\
& \dot{\varphi}_{2}=\omega_{2}+\varepsilon f_{2}\left(\varphi_{1}, \varphi_{2}\right) .
\end{aligned}
$$

Here $f_{1}$ and $f_{2}$ are $2 \pi$-periodic functions in both variables. Also we use a parameter $\varepsilon$ to control the strength of the coupling. For $\varepsilon=0$ we retrieve the format (4). If $T_{1}$ and $T_{2}$ are the respective periods of oscillation, then $\omega_{1}=2 \pi / T_{1}$ and $\omega_{2}=2 \pi / T_{2}$. We first define the Poincaré mapping from the circle $\mathbb{T}^{1}$ to itself and then introduce the rotation number.

\subsubsection{The Poincaré mapping}

If in (5) the size $|\varepsilon|$ of the coupling is not too large, a first-return Poincaré mapping

$$
P: \mathbb{T}^{1} \longrightarrow \mathbb{T}^{1}
$$

is defined, as we shall explain now, also see Figure 3. Without restricting generality we can take the generating circle to be $\mathbb{T}^{1} \times\{0\}$. Baptising $\varphi=\varphi_{1}$ for simplicity, we follow the integral curve from the initial state $\left(\varphi_{1}, \varphi_{2}\right)=(\varphi, 0)$ until $\left(\varphi_{1}, \varphi_{2}\right)=(P(\varphi), 0)$, counting $\bmod 2 \pi \mathbb{Z}$. It is easy to see that $P-\operatorname{Id}$ should be a periodic function in $\varphi$, which gives $P$ the general form

$$
P: \varphi \mapsto \varphi+2 \pi \alpha+\varepsilon f(\varphi),
$$

where $\alpha=\omega_{2} / \omega_{1}$ and where $f$ is a $2 \pi$-periodic function. 
Consideration of the $\mathbb{T}^{1}$-dynamics generated by iteration of $P$ gives a lot of information about the original $\mathbb{T}^{2}$-flow, in particular its asymptotic properties as $t \rightarrow \infty$. For instance, a fixed point attractor of $P$ corresponds to an attracting periodic orbit of the flow which forms a $1: 1$ torus knot as we saw at the end of $\S 1.1$. Similarly a periodic attractor of $P$ of period $q$ corresponds to an attracting periodic orbit of the flow. In general, periodicity will be related to resonance, but to explain this further we need the notion of rotation number.

\subsubsection{Rotation number}

For orientation-preserving homeomorphisms $P: \mathbb{T}^{1} \longrightarrow \mathbb{T}^{1}$ Poincaré has left us the extremely useful concept of rotation number $\varrho(P)$, which describes the average amount of rotation as follows:

$$
\varrho(P)=\frac{1}{2 \pi} \lim _{n \rightarrow \infty} \frac{1}{n}(\tilde{P})^{n}(\varphi) \bmod \mathbb{Z}
$$

Here $\tilde{P}: \mathbb{R} \longrightarrow \mathbb{R}$ is a (non-unique) lift of $P$ which makes the diagram

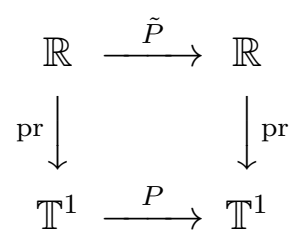

commute, where pr: $\mathbb{R} \longrightarrow \mathbb{T}^{1}$ is the natural projection $\varphi \mapsto \mathrm{e}^{\mathrm{i} \varphi}$. This means that in the formula (8) we do not count modulo $2 \pi$, but keep counting in $\mathbb{R}$.

From $[52,71]$ we quote a number of properties of $\varrho(P)$ :

1. $\varrho(P)$ depends neither on the choice of the lift $\tilde{P}$ nor on the choice of $\varphi$;

2. $\varrho(P)$ is invariant under topological conjugation. This means that if $h$ : $\mathbb{T}^{1} \longrightarrow \mathbb{T}^{1}$ is another orientation-preserving homeomorphism, then

$$
\varrho\left(h P h^{-1}\right)=\varrho(P)
$$

3. If $P: \varphi \mapsto \varphi+2 \pi \alpha$ is a rigid rotation then $\varrho(P)=\alpha \bmod \mathbb{Z}$.

4. $\varrho(P) \in \mathbb{Q}$ precisely when $P$ has a periodic point. Moreover, $\varrho(P)=p / q$ with $p$ and $q$ relatively prime corresponds to a $p: q$ torus knot. 


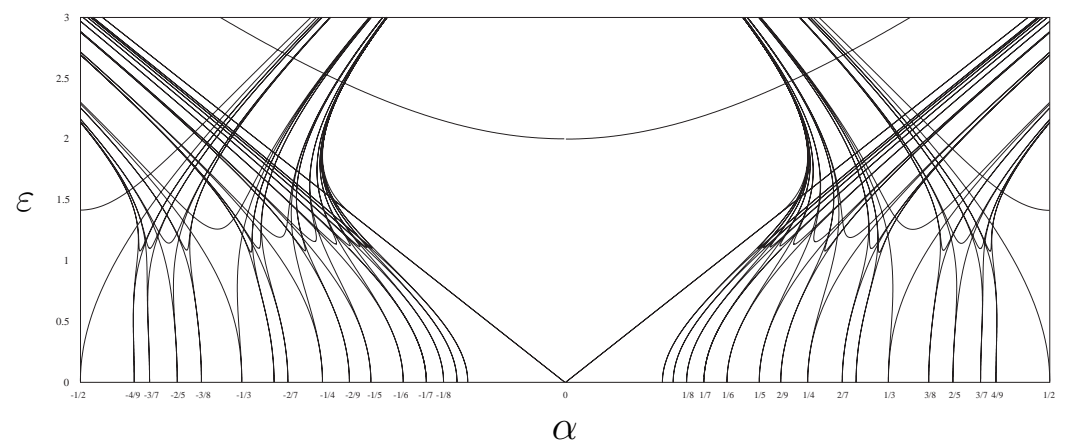

Figure 4: Resonance tongues in the Arnold family (9).

5. If $P$ is of class $C^{2}$ and $\varrho(P)=\alpha$ for $\alpha \in \mathbb{R} \backslash \mathbb{Q}$, then, by a result of Denjoy, the mapping $P$ is topologically conjugated to the rigid rotation $\varphi \mapsto$ $\varphi+2 \pi \alpha$.

Recall that in that case any orbit $\left\{P^{n}(\varphi)\right\}_{n \in \mathbb{Z}}$ forms a dense subset of $\mathbb{T}^{1}$. The corresponding dynamics is called quasi-periodic.

6. If $P$ depends continuously on a parameter, then so does $\varrho(P)$.

\subsubsection{The Arnold family of circle mappings}

A famous example of maps exhibiting resonance is formed by the Arnold family

$$
\mathbb{A}_{\alpha, \varepsilon}: \varphi \mapsto \varphi+2 \pi \alpha+\varepsilon \sin \varphi
$$

of circle mappings. So this is the general format (7) where we chose $f(\varphi)=$ $\sin \varphi .^{1}$

Periodicity. It is instructive to consider the fixed points of (9), given by the equation

$$
\mathbb{A}_{\alpha, \varepsilon}(\varphi)=\varphi
$$

or, equivalently,

$$
\sin \varphi=-\frac{2 \pi \alpha}{\varepsilon}
$$

\footnotetext{
${ }^{1}$ For simplicity we take $|\varepsilon|<1$ which ensures that (9) is a circle diffeomorphism; for $|\varepsilon| \geq 1$ the mapping becomes a circle endomorphism and the current approach breaks down.
} 


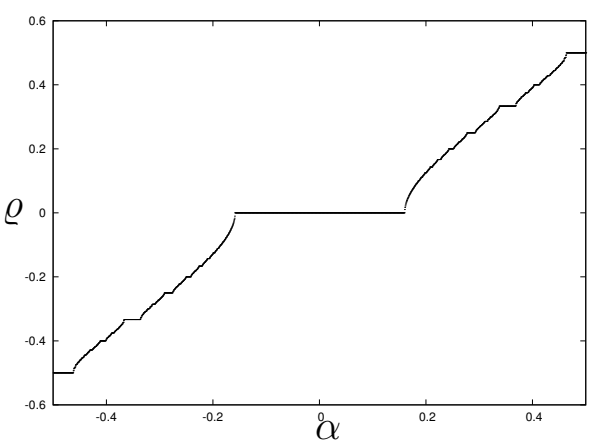

Figure 5: Devil's staircase related to the Arnold family (9). For small $\varepsilon_{0}>0$ the rotation number $\varrho\left(\mathbb{A}_{\alpha, \varepsilon_{0}}\right)$ is depicted as a graph of $\alpha \in[0,1]$.

A brief graphical inspection reveals that $\bmod 2 \pi \mathbb{Z}$ this equation has exactly two solutions for

$$
|\varepsilon|>2 \pi|\alpha| \text {. }
$$

In Figure 4 this region, bounded by the two straight lines $\varepsilon= \pm 2 \pi \alpha$, is depicted for $\varepsilon>0$. It is not hard to see that one of the fixed points is attracting and the other repelling. At the boundary $|\varepsilon|=2 \pi|\alpha|$ these annihilate one another in a saddlenode bifurcation. For the entire region $|\varepsilon| \geq 2 \pi|\alpha|$ one has $\varrho\left(\mathbb{A}_{\alpha, \varepsilon}\right)=0 \bmod \mathbb{Z}$. This region is called the Arnold tongue of rotation number 0 .

From the properties of $\S$ 1.2.2 it follows that, for $(\alpha, \varepsilon)=(p / q, 0)$ with $p$ and $q$ relatively prime, one has $\varrho\left(\mathbb{A}_{\alpha, \varepsilon}\right)=p / q$. One can show that from each $(\alpha, \varepsilon)=$ $(p / q, 0)$ an Arnold tongue emanates, in which for all the parameter points $(\alpha, \varepsilon)$ one has $\varrho\left(\mathbb{A}_{\alpha, \varepsilon}\right)=p / q$, see Figure 4. The 'sharpness', i.e., the order of contact of the boundaries of the $p / q$-tongue at $(\alpha, \varepsilon)=(p / q, 0)$ exactly is of order $q$, see $[1,3,36]$.

Fixing $\varepsilon=\varepsilon_{0}>0$ small, we consider the graph of $\alpha \mapsto \varrho\left(\mathbb{A}_{\alpha, \varepsilon_{0}}\right)$. By another general property of $\S 1.2 .2$, this function is continuous. Moreover, for every rational value $p / q$ it is constant on some plateau, corresponding to the $p / q$-tongue, see Figure 4. The total result is a devil's staircase as depicted in Figure 5.

Quasi-periodicity. In between the tongues the rotation number $\varrho\left(\mathbb{A}_{\alpha, \varepsilon}\right)$ is irrational and by the properties of $\S 1.2 .2$ we know that the corresponding iteration dynamics of $\mathbb{A}$ is quasi-periodic and that each individual orbits densely fills $\mathbb{T}^{1}$. 
Open \& dense versus nowhere dense. In general the $(\alpha, \varepsilon)$-plane of parameters contains a catalogue of the circle dynamics. Again fixing $\varepsilon=\varepsilon_{0}>0$ small, consider the corresponding horizontal line in the $(\alpha, \varepsilon)$-plane of parameters. We witness the following, also see Figure 5 and compare with [36] and references therein. The periodic case corresponds to an open $\&$ dense subset of the line, and the quasi-periodic case to a nowhere dense subset, which in the 1-dimensional situation is a Cantor set.

Diophantic rotation numbers. Quasi-periodicity corresponds to $\varrho=\varrho\left(P_{\alpha, \varepsilon_{0}}\right) \notin$ $\mathbb{Q}$. If we restrict even further to Diophantine $\varrho$ by requiring that for constants $\tau>2$ and $\gamma>0$, for all rationals $p / q$

$$
\left|\varrho-\frac{p}{q}\right| \geq \frac{\gamma}{|q|^{\tau}},
$$

the conjugations of $P_{\alpha, \varepsilon_{0}}$ with the rigid rotation $\varphi \mapsto \varphi+2 \pi \varrho$ can be taken smooth $[1,42]$. The rotation numbers $\varrho$ satisfying (10) form a Cantor subset of the former, which has positive Lebesgue measure, which, by choosing $\gamma=\gamma\left(\varepsilon_{0}\right)=$ $\mathcal{O}\left(\varepsilon_{0}\right)$, can be shown to tend to full measure as $\varepsilon_{0} \rightarrow 0$. A fortiori this holds for the original Cantor set given by $\varrho\left(P_{\alpha, \varepsilon_{0}}\right) \notin \mathbb{Q}$.

Fractal geometry. The Cantor sets under consideration, since they have positive Lebesgue measure, have Hausdorff dimension equal to 1 . Moreover Cantor sets have topological dimension 0 , since they are totally disconnected: every point has arbitrarily small neighbourhoods with empty boundary. The fact that the Hausdorff dimension strictly exceeds the topological dimension is a characterisation of fractals, see page 15 of [66]. So our Cantor sets are fractals. They also show a lot of self-similarity, a property shared with many other fractals.

Beyond the Arnold family (9) . . . The decomposition of the parameter space in an open $\&$ dense set on the one hand, versus a nowhere dense, fractal set of positive measure turns out to be universal, also see [36]. To begin with, any arbitrary smooth (Poincaré) circle mapping of the more general format (7)

$$
P_{\alpha, \varepsilon}: \varphi \mapsto \varphi+2 \pi \alpha+\varepsilon f(\varphi)
$$

turns out to have an array of resonance tongues similar to the Arnold family (9), forming an open \& dense set that corresponds to periodicity, with a fractal complement which is nowhere dense and of positive measure that corresponds to quasiperiodicity. 
The only point of difference with (9) is formed by the exact 'sharpness' of the tongues, which depends on the Fourier coefficients of the function $f$. In particular a tongue at the tip $(\alpha, \varepsilon)=(p / q, 0)$ has transverse boundaries if and only if the $q$ th Fourier coefficient does not vanish.

\subsubsection{Link with resonance}

Returning to the driven oscillator or the two coupled oscillators we now link periodicity of the Poincaré mapping (7) with resonance. For simplicity we keep $|\varepsilon|$ sufficiently small to ensure this mapping to be a diffeomorphism.

As observed in $\S 1.2 .2$ the fact that $(\alpha, \varepsilon)$ belongs to the $p / q$-tongue, i.e., that $\varrho\left(P_{\alpha, \varepsilon}\right)=p / q$, means that the motion takes place on a $p: q$ torus knot. Generically these periodic orbits come in attracting and repelling pairs and the visible motion takes place on such a periodic attractor. In view of our general 'definition' of resonance in that case we say that the oscillators are in $p: q$ resonance, one also speaks of phase-locking or synchronisation. In the case of $1: 1$ resonance sometimes the term entrainment is being used.

If $(\alpha, \varepsilon)$ is outside the tongues, by the Denjoy theory mentioned before, the torus motion takes place on a dense orbit. We also call this torus motion quasi-periodic. In this case from KAM Theory [5, 24, 42] we derive the following. For Diophantine $\varrho=\varrho\left(P_{\alpha, \varepsilon}\right)$, up to a smooth transformation the Poincaré mapping reads

$$
\varphi \mapsto \varphi+2 \pi \varrho
$$

and the two oscillators correspondingly have the familiar decoupled form

$$
\begin{aligned}
& \dot{\varphi}_{1}=\omega_{1} \\
& \dot{\varphi}_{2}=\omega_{2},
\end{aligned}
$$

such that $\varrho\left(P_{\alpha, \varepsilon}\right)=\omega_{2} / \omega_{1}$ for all $\varepsilon$ with $|\varepsilon| \ll 1$. In the parameter space this corresponds to a piece of curve through $(\alpha, \varepsilon)=(\varrho, 0)$, parametrized by $\varepsilon$. We emphasize that this uncountable union of these curves has positive measure.

\subsection{Conclusions and examples}

The literature on resonance phenomena is immense, apart from the references already given, for instance see $[3,42,57,87,90]$ and their bibliographies. For even more references see below. The present point of view models resonant systems 
in terms of dynamical systems depending on parameters, where resonance takes place in a persistent way.

In the parameter space the resonant set is part of the bifurcation set, which forms a catalogue for transitions to various types of dynamics. What we add to the general discussion on this subject is the overall fractal geometry that usually manifests itself in the complement of all the resonances. We now present a couple of examples.

Huygens's clocks. Returning to Huygens's synchronizing clocks we first consider the problem from the torus flow point of view. We have two almost identical oscillators that are weakly coupled. This means that in (4) for the frequencies we have $\omega_{1} \approx \omega_{2}$ and that $|\varepsilon|$ is small. For the Poincaré mapping (7)

$$
P_{\alpha, \epsilon}(\varphi)=\varphi+2 \pi \alpha+\varepsilon f(\varphi)
$$

we only make the assumption that the first Fourier coefficient of $f(\varphi)$ does not vanish, which ensures that the 1/1-tongue boundaries meet transverse at $(\alpha, \varepsilon)=$ $(0,0)$, where the first 0 has to be taken $\bmod \mathbb{Z}$. Compare with Figure 4.

This implies that $(\alpha, \varepsilon)$ belongs to the 1/1-tongue, i.e., that the pendulum clocks are in $1: 1$ resonance, a situation described before as entrainment which is a form of synchronization. This gives a partial explanation of the phenomena discovered by Huygens [60].

\section{Remarks.}

- Note that the 1: 1 resonance of the two clocks could be obtained under quite weak assumptions. If one also wants to understand the phase and antiphase motions, the coupling between the clocks has to be included into the dynamics, compare with $[9,76]$ and references therein.

- For another application of these ideas in terms of circadian rhythms and the response to stimuli see [8]. Here it turns out that next to the $1: 1$ 'entrainment' resonance also certain other resonances have biological significance.

- The above ideas can be largely extended to the case of more than two oscillators. For examples in models for the visual neurocortex see [17, 18]. 
Resonances in the solar system. From ancient times on resonances have been known to occur in the solar system, which are more or less in the spirit of the present section. A well-known example is the orbital 1:2:4 resonance of Jupiter's moons Ganymede, Europa, and Io which was studied by De Sitter [83, 84] using the 'méthodes nouvelles' of Poincaré [75]. The $2: 5$ orbital resonance between Jupiter and Saturn is described by Moser et al. [68, 69, 81]. These and other resonances by certain authors are being held responsable for gaps in the rings of Saturn and in the asteroid belt.

Another type of resonance is the spin-orbit resonance. As an example thereof, the Moon is 'captured by' the Earth in a $1: 1$ resonance: the lunar day with respect to the Earth is (approximately) equal to one month. Similarly Pluto and Charon have caught each other in such a $1: 1$ resonance: as an approximately rigid body the two orbit around the Sun. Interestingly, the planet Mercury is captured in a 3 : 2 spin-orbit resonance around the Sun [50].

\section{Remarks.}

- The spin orbit resonances are explained by tidal forces, for instance, the rotation of the Moon has been slowed down to a standstill by tidal friction brought about largely by the reciprocal tidal forces exerted of Earth and Moon. Similarly the rotation of the Earth in the very long run will be put to a stand still by the tidal forces of mainly the Moon. But probably by that time the Sun has already turned into a red giant ...

- This brings us to the subject of adiabatically changing systems as described and summarized by Arnold [2,3] and which may be used to model such slow changes. ${ }^{2}$ One may perhaps expect that the $3: 2$ spin orbit resonance of Mercury in the very long run, and after quite a number of transitions, will evolve towards another $1: 1$ resonance. This part of nonlinear dynamical systems still is largely unexplored.

\section{Periodically driven oscillators revisited}

We now return to periodically driven oscillators, showing that under certain circumstances exactly the set-up of $\S 1.2$ applies.

\footnotetext{
${ }^{2}$ Mathematical ideas on adiabatic change were used earlier by Rayleigh and Poincaré and by Landau-Lifschitz.
} 


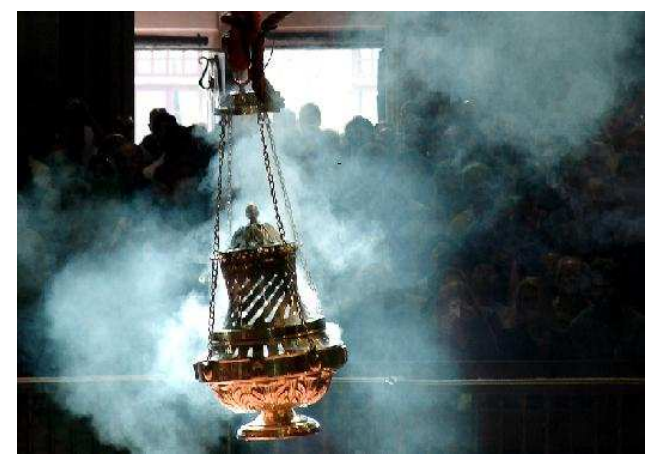

Figure 6: Botafumeiro in Santiago de Compostela.

As a motivating example we discuss the Botafumeiro in the cathedral of Santiago de Compostela, see Figure 6. Here a large incense container is suspended by a pully in the dome where it can swing in the longitudinal direction of the church. A few men pull up the container when it approaches the ground and let go after, thereby creating a periodic forcing and in this way creating a stable motion of exactly twice the period of the forcing.

\subsection{Parametric resonance}

As another model consider the parametrically driven oscillator

$$
\ddot{x}+(a+\varepsilon f(t)) \sin x=0
$$

with $q(t+2 \pi) \equiv q(t)$, see [44]. Here $a$ and $\varepsilon$ are considered as parameters. ${ }^{3}$ For the periodic function $f$ we have studied several examples, namely

$$
\begin{aligned}
f(t) & =\cos t \quad \text { and } \\
& =\cos t+\frac{3}{2} \cos (2 t) \text { and } \\
& =\operatorname{signum}(\cos t),
\end{aligned}
$$

corresponding to the Mathieu case, the Mathieu case modified by a higher harmonic, and the square case, respectively. In this setting the issue is whether the trivial $2 \pi$-periodic solution

$$
x(t) \equiv 0 \equiv \dot{x}(t)
$$

\footnotetext{
${ }^{3}$ For 'historical' reasons we use the letter $a$ instead of $\alpha^{2}$ as we did earlier.
} 


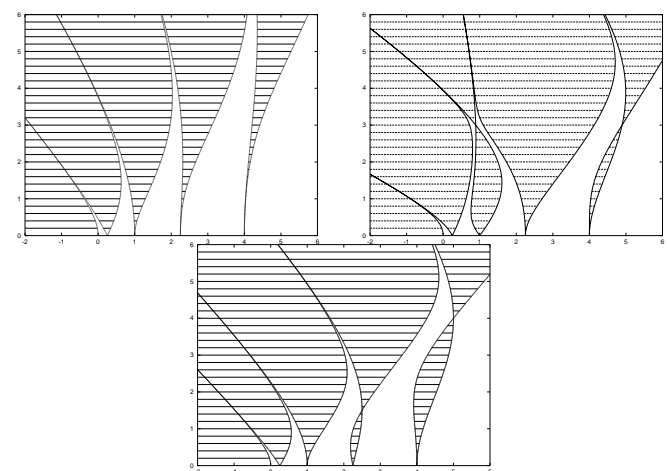

Figure 7: Stability diagrams of Mathieu, modified Mathieu and square case [35].

is elliptic, hence stable, or not. For any of such system (11) from the points

$$
(a, \varepsilon)=\left(\frac{1}{4} k^{2}, 0\right), \quad k=0,1,2, \ldots
$$

tongues emanate in the $(a, \varepsilon)$-plane, where inside the trivial $2 \pi$-periodic solution is hyperbolic, hence unstable. On the tongue boundaries this solution is parabolic. See Figure 7 and compare with [31,35]. We note that this gives rise to a discrete union of tongues, where again the sharpness is governed by the Fourier coefficients of the periodic function $f=f(t)$.

Subharmonics and covering spaces. On the tongue boundaries subharmonic bifurcations occur, see [24, 44, 45] where each bifurcation can be understood in terms of a pitchfork bifurcation on a suitable covering space.

Generally, for the $p: q$ resonance this is constructed as follows. Writing

$$
z=\dot{x}+\mathrm{i} \frac{p}{q} x
$$

where we identify $\mathbb{C} \cong \mathbb{R}^{2}$, the covering map is given by the Van der Pol transformation

$$
\begin{gathered}
\Pi: \mathbb{C} \times \mathbb{R} /(2 \pi q \mathbb{Z}) \longrightarrow \mathbb{C} \times \mathbb{R} /(2 \pi \mathbb{Z}) \\
(\zeta, t) \mapsto\left(\zeta \mathrm{e}^{\mathrm{i} t p / q}, t(\bmod 2 \pi \mathbb{Z})\right)
\end{gathered}
$$



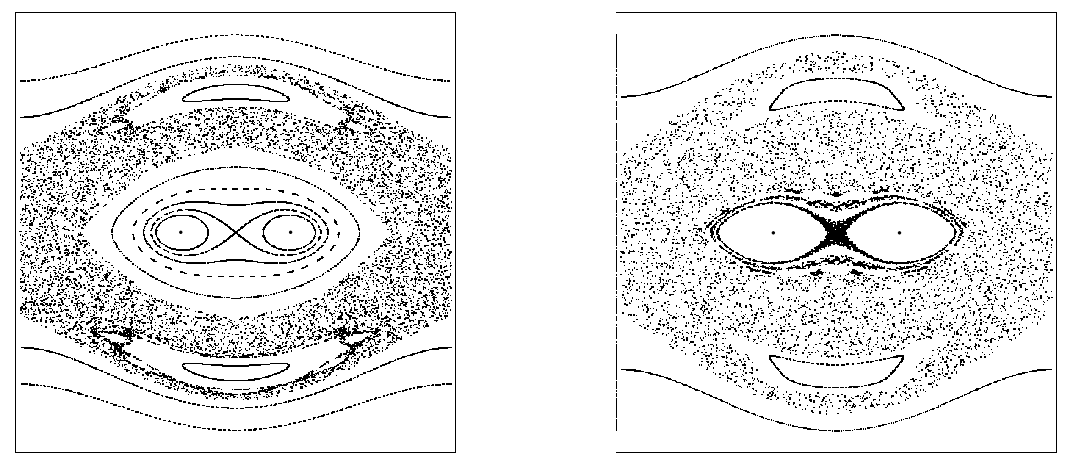

Figure 8: Orbits of the Poincaré mapping of the swing (11).

On the covering space the group $\mathcal{D}$ of decktransformations is generated by

$$
(\zeta, t) \mapsto\left(\zeta \mathrm{e}^{2 \pi p / q}, t-2 \pi\right),
$$

which means that $\Pi \circ T=\Pi$ for any $T \in \mathcal{D} .^{4}$ Note that $\mathcal{D}$ is cyclic of order $q$, i.e., isomorphic to $\mathbb{Z}_{q}$, for details see $[44,45]$. Instead of the original system in $(z, t)$ we pull the system back along $\Pi$, so obtaining a $\mathbb{Z}_{q}$-equivariant system on the $(\zeta, t)$-covering space. In fact this defines a $1: 1$ correspondence of systems and usually it is most convenient to work on the covering space. Generally on the covering space equivariant Singularity Theory can be practised, see $[19,55,56]$ and references therein, as well as equivariant KAM Theory [14, 22, 23, 24, 30]. We refer to Appendix A.3 for further details.

In the present case this construction is only needed for $p / q=k / 2$. The strongest of these resonances occurs inside the tongue labeled by $k=1$, where a cylinder with $\mathbb{Z}_{2}$-symmetry is the double cover of a Möbius strip, for a remark in this direction see [57]. This is the setting for the simplest subharmonic bifurcation, namely a period doubling bifurcation: inside the tongue the trivial $2 \pi$-periodic solution is unstable but a stable periodic orbit occurs of period $4 \pi$. At the boundary a period-doubling bifurcation takes place. The corresponding $\mathbb{Z}_{2}$-equivariant bifurcation on the covering space exactly is the pitchfork. The ensuing period-doubled periodic motion is exactly the one that occurs in the Botafumeiro example. See Figure 8 for 'phase portaits' of the Poincaré mapping, left for $\varepsilon=0.25$ and right for $\varepsilon=0.40$

\section{Remarks.}

\footnotetext{
${ }^{4}$ The co-ordinates $(\zeta, t)$ sometimes also are called co-rotating. Also think of the Lagrangean variation of constants.
} 
- The geometric complexity of the individual tongues in Figure 7 can be described by Singularity Theory; in fact it turns out that we are dealing with type $\mathbb{A}_{2 k-1}$, see $[35,44]$.

- The parametric $1: 2$ resonance sometimes also is called the parametric roll. By this mechanism ships have been known to capsize ...

- In Figure 8 also invariant circles can be witnessed. KAM Theory, as discussed before, in particular an application of Moser's Twist Theorem [67], shows that the union of such invariant circles carrying quasi-periodic dynamics has positive measure.

- In both cases the cloud of points ${ }^{5}$ is formed by just one or two orbits under the iteration of the Poincare mapping. These clouds are associated to homoclinic orbits related to the upside down unstable periodic solution, which gives rise to horseshoes. Therefore such an orbit is chaotic since it has positive topological entropy, see [42] and references therein. A classical conjecture is that the cloud densely fills a subset of the plane of positive Lebesgue measure on which the Poincaré mapping is ergodic [4]. ${ }^{6}$

\subsection{The Hill-Schrödinger equation}

Another famous equation is a linearized version of (11) where the forcing term is quasi-periodic in $t$ :

$$
\ddot{x}+(a+\varepsilon f(t)) x=0,
$$

where now $f(t)=F\left(\omega_{1} t, \omega_{2} t, \ldots, \omega_{n} t\right)$ for a function $F: \mathbb{T}^{n} \rightarrow \mathbb{R}$, see [32, 53, 70]. As in the case of $\S 1.2$ the countable union of tongues again becomes open $\&$ dense and separated by a nowhere dense set of positive measure, determined by Diophantine conditions. The geometry of the individual tongues for small $|\varepsilon|$ is exactly as in the periodic case. For larger values of $|\varepsilon|$ the situation is more complicated also involving non-reducible quasi-periodic tori, compare with [34].

The equation (12) happens to be the eigenvalue equation of the 1-dimensional Schrödinger operator with quasi-periodic potential. We here sketch how our geometric approach fits within the corresponding operator theory. This operator reads

$$
\left(H_{\varepsilon f} x\right)(t)=-\ddot{x}(t)-\varepsilon f(t) x(t)
$$

${ }^{5}$ Colloquially often referred to as 'chaotic sea'.

${ }^{6}$ Also known as the metric entropy conjecture. 


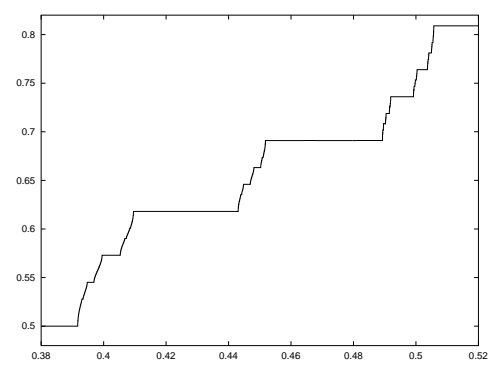

Figure 9: Devil's staircase in the Schrödinger equation with quasi-periodic potential: $\omega_{1}=1$ and $\omega_{2}=\frac{1}{2}(\sqrt{5}-1)$, see [32].

with potential $\varepsilon f$; it acts on wave functions $x=x(t) \in L^{2}(\mathbb{R})$.

We like to note that in the corresponding literature usually the value of $\varepsilon=\varepsilon_{0} \neq 0$ is fixed and the intersection of the horizontal line $\varepsilon=\varepsilon_{0}$ with a tongue is referred to as gap: it is a gap in the spectrum of the Schrödinger operator (13). The approach with tongues and the results of [32] regarding the $\mathbb{A}_{2 k-1}-$ singularity therefore leads to a generic gap closing theory.

\section{Remarks.}

- In the context of Schrödinger operators the letters are chosen somewhat differently. In particular, instead of $x(t)$ one often considers $u(x)$, which gives this theory a spatial interpretation. Also instead of $\varepsilon f(t)$ one uses $V(x)$, compare with [70].

- For a fixed value $\varepsilon=\varepsilon_{0}$ the Diophantine Cantor set leads to Cantor spectrum. The total picture is illustrated in the devil's staircase of Figure 9, where we took $n=2, \omega_{1}=1$ and $\omega_{2}=\frac{1}{2}(\sqrt{5}-1)$. The rotation number $\varrho$ is defined almost as before [32] as a function of $a$.

- The nonlinear equation

$$
\ddot{x}+(a+\varepsilon f(t)) \sin x=0,
$$

with $q$ quasi-periodic is dealt with in [22]. In comparison with the case of periodic $f$ the averaged, approximating situation, is identical. However, the infinite number of resonances and the Cantorization we saw before leads to an infinite regress of the bifurcation scenarios. For this use was made of equivariant Hamiltonian KAM Theory on a suitable covering space [24, 30]. As a consequence the resonant set becomes residual and the quasi-periodic set meagre. Compare this with $[7,46,47,48]$ in the dissipative case. 


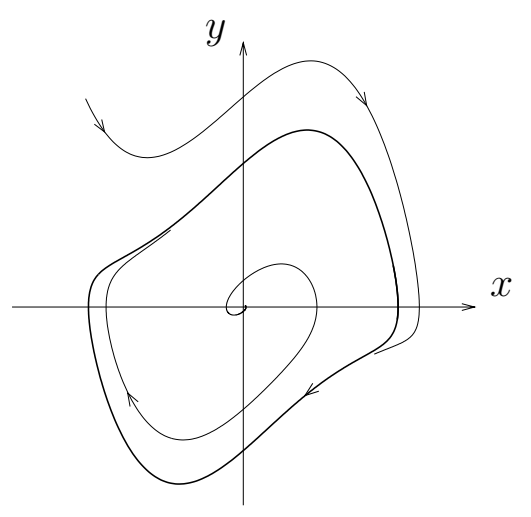

Figure 10: Phase portrait of the free Van der Pol oscillator [42].

\subsection{Driven and coupled Van der Pol-like oscillators}

The examples of the driven oscillator in $\S 1.1$ were based on approximations of the damped pendulum, the free oscillation of which always tends to the lower equilibrium $x=0, \dot{x}=0$. Our present interest is formed by Van der Pol-like oscillators that for $|x|$ and $|\dot{x}|$ sufficiently small have negative damping, for this approach compare with $[24,42]$. Such oscillators are known to occur in electronics $[77,78,80]$.

Therefore our starting point is the periodically driven Van der Pol oscillator in a slightly more general form

$$
\ddot{x}=-\alpha^{2} x-c \dot{x}-a(x, \dot{x})+\varepsilon f(x, \dot{x}, t ; \varepsilon),
$$

where the function $q$ is $2 \pi$-periodic in the time $t$. Van der Pol originally considered $a(x, \dot{x})=b x^{2} \dot{x}$ and $f(x, \dot{x}, t ; \varepsilon)=\sin t$. We here assume that $a$ and $q$ are sufficiently smooth, say of class $C^{\infty}$ or real-analytic. The important restriction on $a$ is that the corresponding free oscillator has a hyperbolic attractor. For a phase portrait in the $(x, \dot{x})$-plane, see Figure 10. Passing to the system form

$$
\begin{aligned}
\dot{x} & =y \\
\dot{y} & =-\alpha^{2} x-c y-a(x, y)+\varepsilon F(x, y, z ; \varepsilon) \\
\dot{z} & =1,
\end{aligned}
$$

as before, we get a 3 -dimensional state space $\mathbb{R}^{2} \times \mathbb{T}^{1}=\{(x, y), z\}$, so with angular variable $z$. Let us denote the corresponding vector field by $X_{\alpha, \varepsilon}$. 
This brings us back to the general setting of a 2-torus flow, with two phase angles $\varphi_{1}, \varphi_{2}$, e.g., with $\varphi_{1}$ the phase of the free oscillator, i.e., its time parametrization scaled to period $2 \pi$, and $\varphi_{2}=z$. For $\varepsilon=0$ we so obtain

$$
\begin{aligned}
& \dot{\varphi}_{1}=\omega_{1} \\
& \dot{\varphi}_{2}=\omega_{2},
\end{aligned}
$$

which is of the familiar format (4). From here the theory of $\S 1.2$ applies in al its complexity, with in the parameter space an open \& dense, countable union of resonances and a fractal set of positive measure regarding quasi-periodicity.

Similar results hold for $n$ coupled Van der Pol type oscillators, now with state space $\mathbb{T}^{n}$, the cartesian product of $n$ copies of $\mathbb{T}^{1}$. Next to periodic and quasiperiodic motion, now also chaotic motions occur, see [42] and references therein.

\section{Universal studies}

Instead of studying classes of driven or coupled oscillators we now turn to a few universal cases of 'generic' bifurcations. The first of these is the Hopf-NeimarkSacker bifurcation for diffeomorphisms, which has occurrence codimension 1. This means that the bifurcation occurs persistently in generic 1-parameter families. However, the open $\&$ dense occurrence of countably many resonances and the complementary fractal geometry of positive measure in the bifurcation set are only persistent in generic 2-parameter families. A second bifurcation we study is the Hopf saddle-node bifurcation for diffeomorphisms where we use 3 parameters for describing the persistent complexity of the bifurcation set.

\subsection{The Hopf-Neĭmark-Sacker bifurcation}

We start with the Hopf-Nermark-Sacker bifurcation for diffeomorphisms, but also discuss certain consequences for systems of differential equations. As an example to illustrate our ideas consider the following Duffing-Van der Pol-Liénard type driven oscillator

$$
\ddot{x}+\left(\nu_{1}+\nu_{3} x^{2}\right) \dot{x}+\nu_{2} x+\nu_{4} x^{3}+x^{5}=\varepsilon\left(1+x^{6}\right) \sin t,
$$

the coefficients of which can be considered as parameters. Note that for the free oscillator at $\nu_{1}=0$ the eigenvalues of the linear part at $(x, \dot{x})=(0,0)$ cross the 


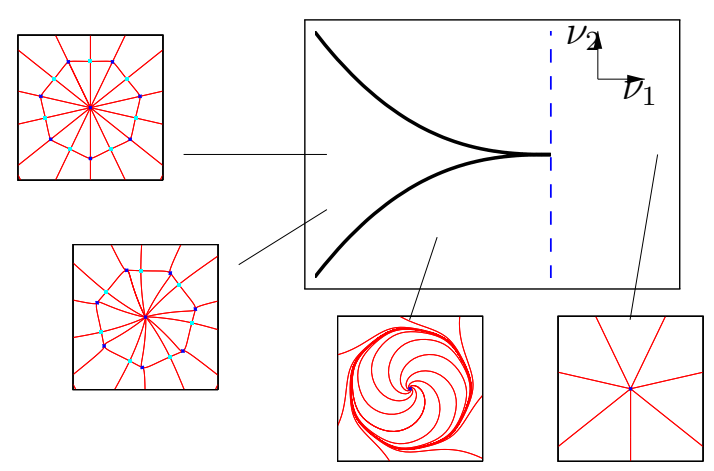

Figure 11: Tongue in nondegenerate resonance $q=7$ of the Hopf-Ner̆markSacker bifurcation $[19,25,26,28]$.

imaginary axis at $\pm \mathrm{i} \sqrt{\nu_{2}}$. Excluding the strong resonances where $\sqrt{\nu_{2}}=p / q$ for $p$ and $q$ relatively prime with $q \leq 5$ and assuming that the constants $\nu_{3}$ and $\nu_{4}$ are generically chosen, the organization of the $\left(\nu_{1}, \nu_{2}\right)$-plane, for $\nu_{1}$ consists of an an open $\&$ dense union of countably many resonance tongues separated by a nowhere dense set of positive measure. This situation is comparable to the Arnold family (9) and Figure 4, at least for large values of $q$.

\subsubsection{The nondegerate case}

The general set-up just considers a mapping

$$
P: \mathbb{R}^{2} \longrightarrow \mathbb{R}^{2}
$$

around a fixed point, say $P(0)=0$, where the eigenvalues of the linear part read $\mathrm{e}^{\nu_{1} \pm 2 \pi \mathrm{i} \sqrt{\nu_{2}}}$ with $\left|\nu_{1}\right|$ small. We consider a fixed resonance $\left(\nu_{1}, \nu_{2}\right)=\left(0, p^{2} / q^{2}\right)$ with $p$ and $q$ relatively prime, where we will need 2 parameters to versally unfold the linear part $[3,24]$. Our main interest is with the periodic points of period $q$, so in solving the equation

$$
P^{q}(x, y)=(x, y) .
$$

The zeroes of $P^{q}-\mathrm{Id}$ are studied by a Lyapunov-Schmidt reduction, cf. Appendix A.1. This leads to a $\mathbb{Z}_{q}$-equivariant family of functions

$$
G_{\mu}(z)=z B_{\mu}(u)+C_{\mu} \bar{z}^{q-1}+\mathcal{O}\left(|z|^{q}\right)
$$

where $z$ is an appropriate complex variable and where $B_{\mu}$ is a polynomial in $u=$ $|z|^{2}$ of degree less than $(q-1) / 2$. We study the corresponding discriminant set 
given by

$$
G_{\mu}(z)=0 \text { and } \operatorname{det} D_{z} G_{\mu}(z)=0 .
$$

Here $\mu$ is an unfolding-multiparameter detuning the resonance at hand. The way to study this discriminant set is by $\mathbb{Z}_{q}$-equivariant contact equivalence $[19,25,26$, $27,28]$. In the present non-degenerate case (16) can be reduced to the polynomial normal form

$$
G_{\sigma}^{\mathrm{NF}}(z)=z\left(\sigma+|z|^{2}\right)+\bar{z}^{q-1}
$$

for a complex parameter $\sigma$. See also Appendix A.2, in particular Theorem 2. In general this set turns out to be a 'tongue' ending in a cusp of sharpness $(q-2) / 2$, which is part of a familiar bifurcation diagram with two periodic orbits of period $q$ inside that annihilate one another at the tongue boundaries in a saddle-node or fold bifurcation [3].

See Figure 11 which is embedded in the context of the equation (14), of which $P$ is a Poincaré mapping. Here the dynamics of $P$ also has been described in terms of a Poincaré-Takens interpolating normal form approximation, e.g., see [13, 44, 45, 88] and Appendix A.3.

Globally a countable union of such cusps is separated by a nowhere dense set of positive measure, corresponding to invariant circles with Diophantine rotation number. As before, see Figure 4, the latter set contains the fractal geometry.

\section{Remarks.}

- The above results, summarized from $[19,25,26,28]$, are mainly obtained by $\mathbb{Z}_{q}$-equivariant Singularity Theory.

- The strong resonances with $q=1,2,3$ and 4 form a completely different story where the Singularity Theory is far more involved $[3,88]$. Still, since the higher order resonances accumulate at the boundaries, there is fractal geometry around, always of positive measure.

- Regarding structural stability of unfoldings of $P$ as in (15) under topological conjugation, all hopes had already disappeared since [64]. 


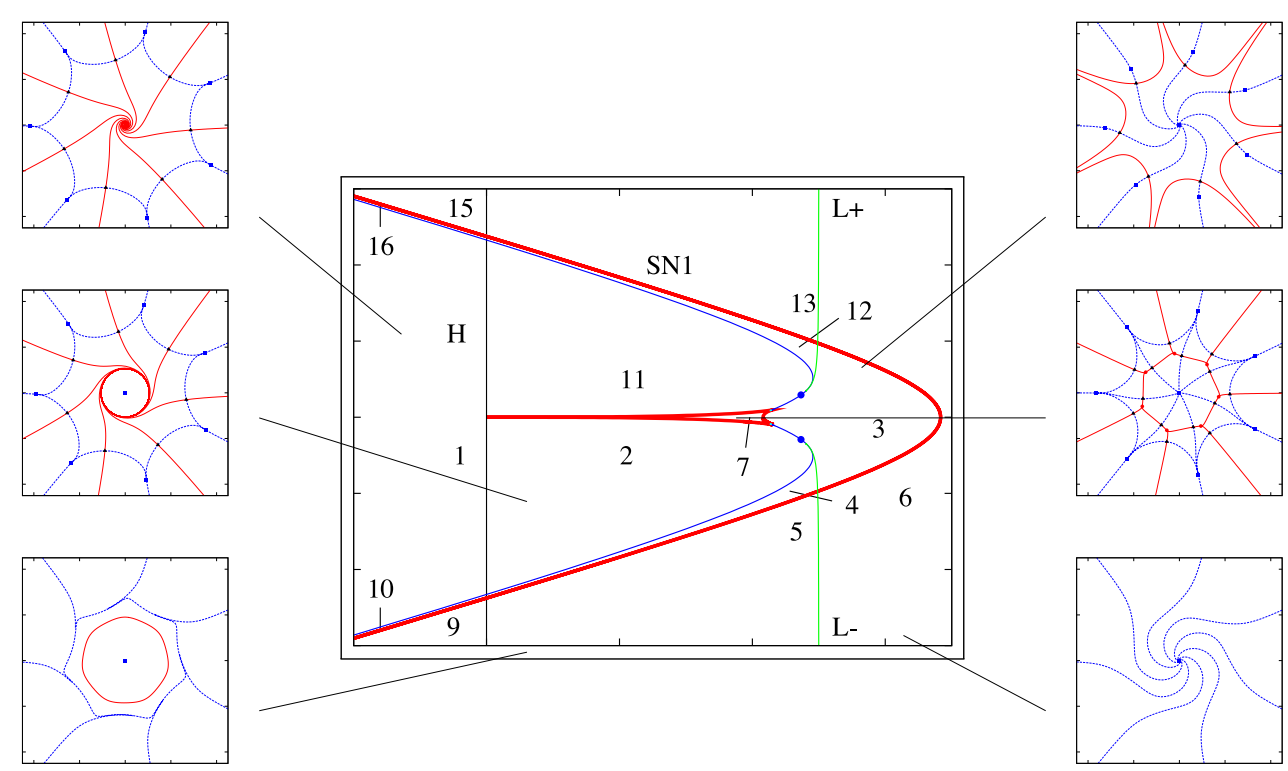

Figure 12: Two-dimensionsional tomography in a mildly degenerate resonance $q=7$ of the Hopf-Neĭmark-Sacker bifurcation [19, 25, 26, 28].

\subsubsection{A mildly degenerate case}

In the mildly degenerate case the 'Hopf' coefficient in the previous example vanishes and is introduced as an extra parameter. This leads to another normal form

$$
G_{\sigma, \tau}^{\mathrm{NF}}(z)=z\left(\sigma+\tau|z|^{2}+|z|^{4}\right)+\bar{z}^{q-1},
$$

which is now parametrized over $\mathbb{C}^{2} \cong \mathbb{R}^{4}$, hence of codimension 4. Again, cf. Appendix A.2, in particular Theorem 2. As before $[19,27]$ the normal form (17) is structurally stable under $\mathbb{Z}_{q}$-equivariant contact equivalence. Here the Singularity Theory is more complex, involving folds, cusps, a swallowtail and a Whitney umbrella. The complete bifurcation diagram is more involved, even at the level of Poincaré-Takens normal form vector field approximations. In Figure 12, again for the case $q=7$, a 2-dimensional tomography is shown of the bifurcation set, in which still a tongue-like structure can be discerned, for more details see [26, 28] where also 3-dimensional tomographies are presented.

To illustrate a mildly degenerate case of the Hopf-Neĭmark-Sacker bifurcation one may well consider the preceding Duffing-Van der Pol-Liénard type driven oscillator (14) where we need all four parameters. 


\subsubsection{Concluding remarks}

For both cases of the Hopf-Neĭmark-Sacker bifurcation we have a good grip on the part of the bifurcation set that governs the number of periodic points. The full bifurcation set is far more involved and the corresponding dynamics is described only at the level of Poincaré-Takens normal-form vector fields [13, 26, 28]. We note that homo- and heteroclinic phenomena occur at a flat distance in terms of the bifurcation parameters $[33,41,74]$.

\subsection{The Hopf saddle-node bifurcation for diffeomorphisms}

As a continuation of the above programme, we now consider the Hopf saddlenode (or fold Hopf) bifurcation for diffeomorphisms [38, 39, 40], in which the central singularity is a fixed point of a 3 -dimensional diffeomorphism, such that the eigenvalues of the linear part at bifurcation are 1 and $\mathrm{e}^{2 \pi \mathrm{i} \alpha}$, where

$$
\mathrm{e}^{2 \pi n \mathrm{i} \alpha} \neq 1 \quad \text { for } n=1,2,3 \text { and } 4,
$$

so excluding strong resonances as in the Hopf-Nel̆mark-Sacker case of $\S 3.1$. The Hopf saddle-node bifurcation for flows is well-known [43, 57], especially because of the subordinate Hopf-Neĭmark-Sacker and Šilnikov homoclinic bifurcation. Our main interest is how the Hopf-Nermark-Sacker bifurcation is being changed into one of the simplest quasi-periodic bifurcations near a $2: 5$ resonance.

\subsubsection{From vector fields to mappings}

The linear part of the vector field at bifurcation has eigenvalues 0 and $\pm \mathrm{i} \alpha$. This linear part generates an axial symmetry that in a normal form procedure can be pushed stepwise over the entire Taylor series, see [13] and references. This makes it possible to first consider axially symmetric systems, that turn out to be topologically determined by their 3rd-order truncation given by

$$
\begin{aligned}
\dot{w} & =\left(-\beta_{2}+\mathrm{i} \alpha\right) w-a w z-w z^{2} \\
\dot{z} & =-\beta_{1}-s w \bar{w}-z^{2}
\end{aligned}
$$

where $w \in \mathbb{C}$ and $z \in \mathbb{R}$ and where $\beta_{1}$ and $\beta_{2}$ are unfolding parameters [57,61]. A scaling $\beta_{1}=\gamma^{2}, \beta_{2}=\gamma^{2} \mu$ leads to a vector field

$$
Y_{\gamma, \mu, \alpha}(z, w)=\left(\begin{array}{c}
(-\gamma \mu+2 \pi \mathrm{i} \alpha / \gamma) w-a w z-\gamma w z^{2} \\
1-z^{2}-|w|^{2}
\end{array}\right)
$$




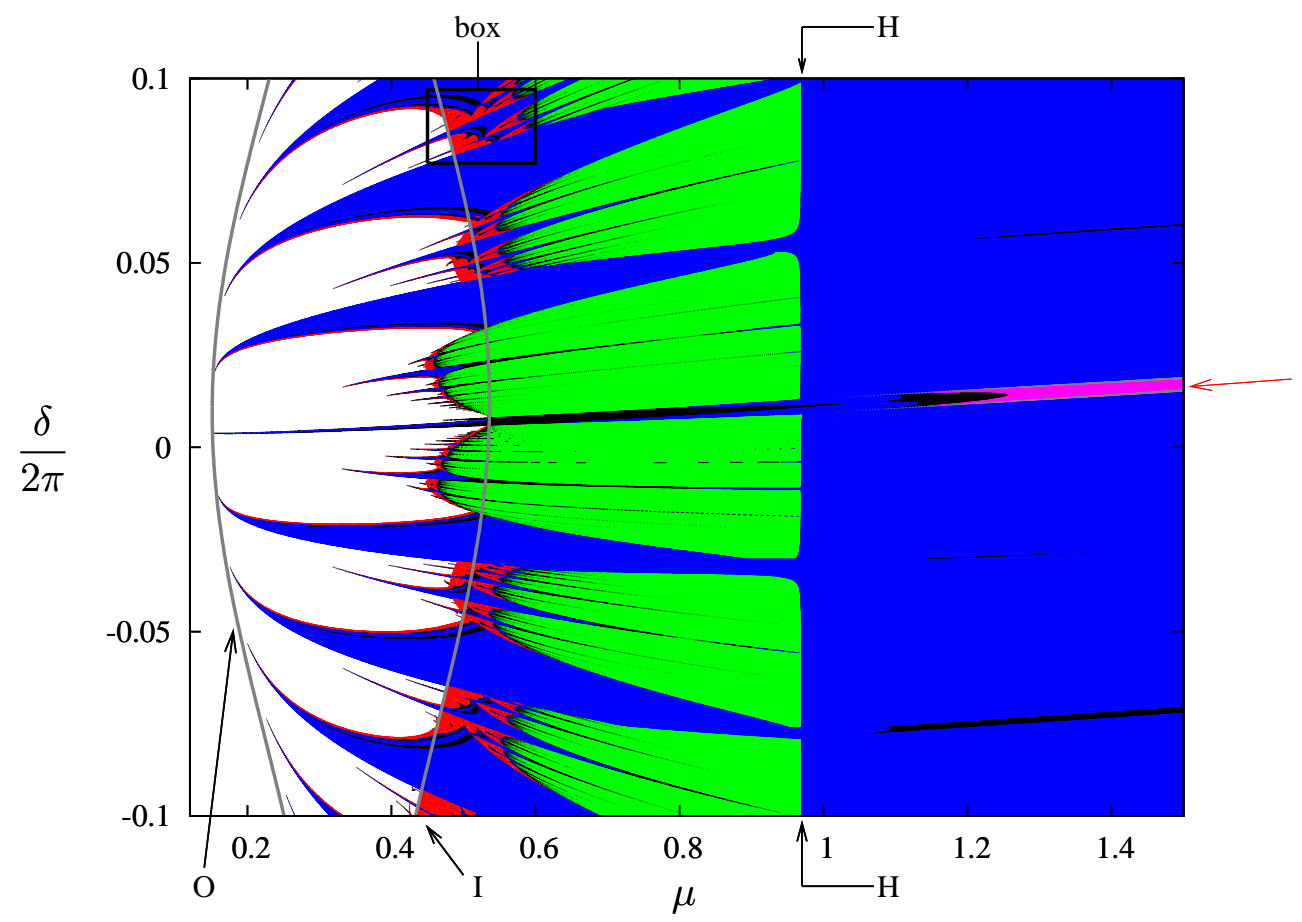

Figure 13: Lyapunov diagram of $G$ in the $(\mu, \delta /(2 \pi))$-parameter plane. The colors correspond to distinct classes of attractors of $G[38,40]$. See table 1 for the color coding.

From this an axially symmetric map

$$
\left(\begin{array}{c}
w \\
z
\end{array}\right) \mapsto\left(\begin{array}{c}
\mathrm{e}^{2 \pi \mathrm{i} \alpha} w\left[1-\gamma\left(\gamma \mu+a z+\gamma z^{2}\right)\right] \\
z-\gamma\left(-1+|w|^{2}+z^{2}\right)
\end{array}\right)
$$

is obtained in a kind of Euler step. To study a 2: 5 resonance we take $\alpha_{0}=2 / 5$, writing $\alpha=\alpha_{0}+\gamma \delta$, and perturb to

$$
G_{\mu, \delta}:\left(\begin{array}{c}
w \\
z
\end{array}\right) \mapsto\left(\begin{array}{c}
\mathrm{e}^{\mathrm{i} \alpha} w\left[1-\gamma\left(\gamma \mu+a z+\gamma z^{2}\right)\right] \\
z-\gamma\left(-1+|w|^{2}+z^{2}\right)
\end{array}\right)+\left(\begin{array}{c}
\gamma^{3}\left(\varepsilon_{1} \bar{w}^{4}+\varepsilon_{2} z^{4}\right) \\
\gamma^{4} \varepsilon_{3} \operatorname{Re} w^{5}
\end{array}\right)
$$

by adding axially non-symmetric order $r$ resonant terms

$$
\bar{w}^{4} \frac{\partial}{\partial w} \text { and } \operatorname{Re} w^{5} \frac{\partial}{\partial z} \text {. }
$$

The scaling parameter $\gamma$ and the other constants are fixed suitably. 


\begin{tabular}{|lll|}
\hline color & Lyapunov exponents & attractor type \\
\hline red & $\ell_{1}>0=\ell_{2}>\ell_{3}$ & strange attractor \\
yellow & $\ell_{1}>0>\ell_{2}>\ell_{3}$ & strange attractor \\
blue & $\ell_{1}=0>\ell_{2}=\ell_{3}$ & invariant circle of focus type \\
green & $\ell_{1}=\ell_{2}=0>\ell_{3}$ & invariant 2-torus \\
black & $\ell_{1}=0>\ell_{2}>\ell_{3}$ & invariant circle of node type \\
grey & $0>\ell_{1}>\ell_{2}=\ell_{3}$ & fixed point of focus type \\
fuchsia & $0>\ell_{1}=\ell_{2} \geq \ell_{3}$ & fixed point of focus type \\
pale blue & $0>\ell_{1}>\ell_{2}>\ell_{3}$ & fixed point of node type \\
white & & no attractor detected \\
\hline
\end{tabular}

Table 1: Legend of the color coding for Figure 13, see $[38,40]$. The attractors are classified by means of the Lyapunov exponents $\left(\ell_{1}, \ell_{2}, \ell_{3}\right)$.

\subsubsection{In the product of state space and parameter space}

In Figure 13 a Lyapunov diagram is depicted in the parameter plane of the mapping family $G$. Table 1 contains the corresponding color code. In Figure 14 we show the dynamics corresponding to two values of $(\mu, \delta / 2 \pi)$. Let us discuss these numerical data.

The parameter space. On the right-hand-side of the figure this method detects an attracting invariant circle of focus type (blue). In the gaps larger resonances are visible, compare with Figure 4 for a fixed value of $\varepsilon$. Moving to the left, in the neighbourhood of the line indicated by $\mathrm{H}$ a quasi-periodic Hopf bifurcation occurs from a circle attractor to a 2-torus attractor (green). Also here the parameter space is interspersed with a resonance web of which the larger lines are visible. The remaining features, among other things, indicate invariant tori and strange attractors of various types and also more invariant circles.

The state space. The upper two figures of Figure 14 show an invariant circle, once seen from the $z$-direction and once from some $w$-direction. The lower two figures indicate how this circle has become a strange attractor, from the same two points of view. 

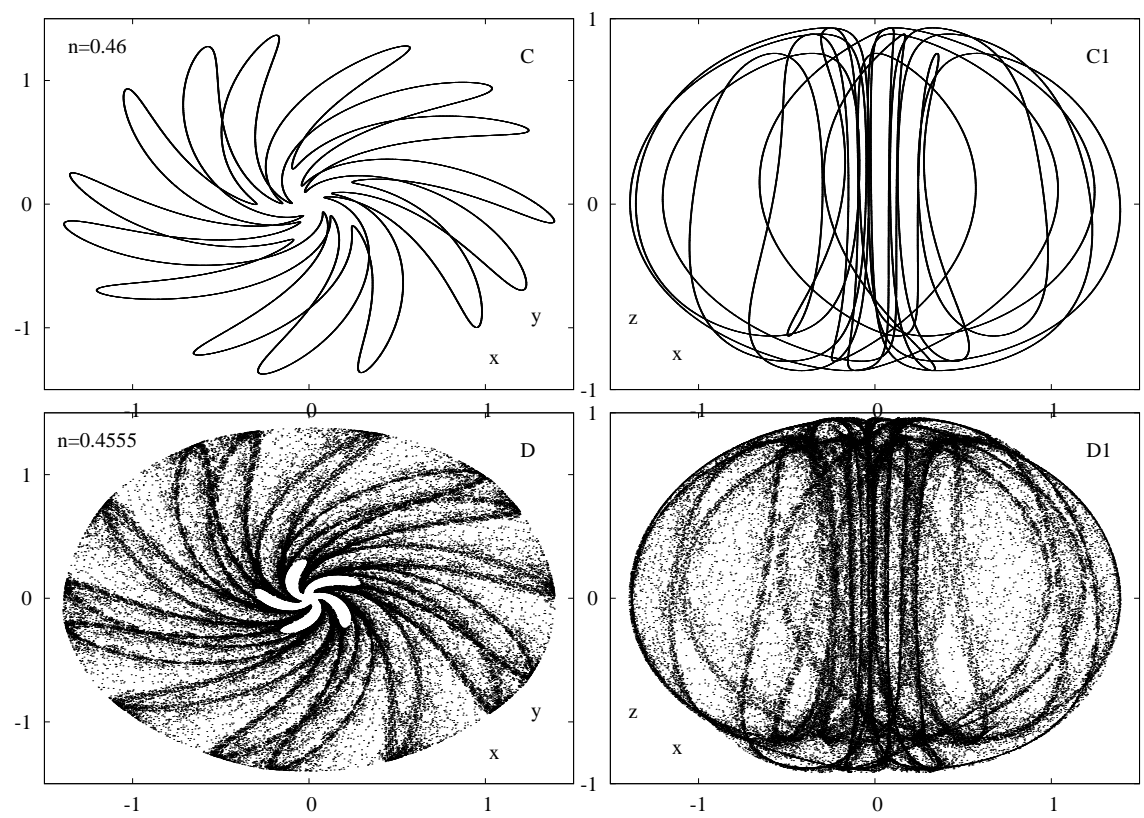

Figure 14: Quasi-periodic (top) and strange attractor (bottom) in the Hopf saddlenode bifurcation for mappings, as seen from two different angles (left and right) $[38,40]$.

Part of these results can be justified mathematically, as seen from the Perturbation Theory point of view. The invariant circles all have one Lyapunov exponent equal to 0 and these are quasi-periodic, perturbations of closed integral curves of a vector field (averaging) approximation, whence their existence can be proven by KAM Theory $[5,30]$. A similar statement can be made about the 2-tori with two Lyapunov exponents equal to 0 . In fact the transition is a quasi-periodic Hopf bifurcation as discussed by Broer et al. [10, 11, 24, 42].

By the same references, this also holds for the quasi-periodic invariant circle in the upper half of Figure 14. The lower half of this figure is conjectured to show a quasi-periodic Hénon-like attractor, which is the closure of the unstable manifold of an unstable quasi-periodic invariant circle. This is the previous quasiperiodic circle that has become unstable through a quasi-periodic saddle-node bifurcation [11]. For this kind of strange attractor the mathematical background theory largely fails, so the results must remain experimental; for indications in this direction however see [40] and references therein.

For a detailed, computer-assisted bifurcation analysis of the $2: 5$ resonance 'bub- 
ble' we refer to [38]. Compare with earlier work of Chenciner [46, 47, 48]. We like to note that the family of mappings $G$ forms a concrete model for the Ruelle-Takens scenario regarding the onset of turbulence. In fact it also illustrates how the earlier scenario of Hopf-Landau-Lifschitz is also included: the present multi-parameter set-up unifies both approaches. For details and background see $[24,42,58,59,62,63,65,79]$.

Resonance and fractal geometry. Interestingly, the blue colors right and left correspond to quasi-periodic circle attractors. The fact that the corresponding regions of the plane look like open sets is misleading. In reality these are meagre sets, dense veined by the residual sets associated to periodicity. These details are just too fine to be detected by the computational precision used.

Particularly in the latter case, in the left half of the diagram, we are dealing with the Arnold resonance web, for a detailed analysis see [39].

\section{Conclusions}

We discuss a number of consequences of the present paper in terms of modelling of increasing complexity.

\section{1 'Next cases'}

The Hopf saddle-node bifurcation for maps, see $\S 3.2$, can be viewed as a 'next case' in the systematic study of bifurcations as compared to, e.g., [57, 61] and many others. The nowhere dense part of parameter space, since it lacks interior points is somewhat problematic to penetrate by numerical continuation methods. Nevertheless, from the 'physical' point of view, this part surely is visible when its measure is positive or, as in the present examples, even close to full measure. Needless to say that this observation already holds for the Hopf-Neĭmark-Sacker bifurcation as described in $\S 3.1$.

Other 'next cases' are formed by the quasi-periodic bifurcations which is a joint application of Kolmogorov-Arnold-Moser Theory [5, 11, 24, 29, 42] and Singularity Theory [54, 55, 56, 89]. For overviews see [12, 49, 91]. The quasi-periodic bifurcations are inspired by the classical ones in which equilibria or periodic orbits are replaced by quasi-periodic tori. As an example, in the Hopf saddle node of $\S 3.2$ we met quasi-periodic Hopf bifurcation for mappings from circles to a 
2-tori in a subordinate way. Here we witness a global geometry inspired by the classical Hopf bifurcation, which concerns the quasi-periodic dynamics associated to the fractal geometry in the parameter space, compare with Figure 13. The gaps or tongues in between concern the resonances inside, within which we notice a further 'fractalization' or 'Cantorization'.

A similar 'next case' in complexity is given by the parametrically forced Lagrange top [21, 29], in which a quasi-periodic Hamiltonian Hopf bifurcation occurs. Indeed, we recall from [51] that in the Lagrange top a Hamiltonian Hopf bifurcation occurs, the geometry of which involves a swallowtail catastrophy. By the periodic forcing this geometry is 'Cantorized' yielding countably many tongues with fractal geometry in between.

\section{Remarks.}

- As said before, in cases with infinite regress the fractal complement is a meagre set which has positive measure. Simon [82] describes a similar situation for 1-dimensional Schrödinger operators. Also see [6].

- It is an interesting property of the real numbers to allow for this kind of dichotomy in measure and topology, compare with Oxtoby [72]. Interestingly, although these properties in the first half of the 20th century were investigated for theoretical reasons, they here naturally show up in the context of resonances and spectra.

\subsection{Modelling}

We like to note that our investigations on the Hopf saddle-node bifurcation for mappings were inspired by climate models [16, 37, 85], where in about 80 dimensional Galerkin projections of PDE models such bifurcations were detected in 3-dimensional center manifolds.

Generally speaking there exists a large-scale programme of modelling in terms of dynamical systems depending on parameters, with applications varying from climate research to mathematical physics and biological cell systems. These models often are high-dimensional and their complexity is partly explained by mechanisms of the present paper, also see $[42,79,91]$. In general such models exhibit the coexistence of periodicity (including resonance), quasi-periodicity and chaos, best observed in the product of state- and parameter space. 


\section{Acknowledgements}

The author thanks Konstantinos Efstathiou, Aernout van Enter and Ferdinand Verhulst for their help in the preparation of this paper.

\section{References}

[1] V.I. Arnol'd, Small divisors I: On mappings of the circle onto itself. American Mathemathical Society Translations, Ser.2 46 (1965) 213-284

[2] V.I. Arnold, Mathematical Methods of Classical Mechanics. GTM 60 Springer 1978

[3] V.I. Arnold, Geometrical Methods in the Theory of Ordinary Differential Equations. Springer 1983

[4] V.I. Arnold and A. Avez, Probèmes Ergodiques de la Mécanique classique. Gauthier-Villars 1967; Ergodic problems of classical mechanics. Benjamin 1968.

[5] V.I. Arnol'd, V.V. Kozlov and A.I. Neishtadt, Mathematical Aspects of Classical and Celestial Mechanics. In: V.I. Arnol'd (ed.) Dynamical Systems III. Springer 1988.

[6] A. Avila and D. Damanik, Generic singular spectrum for ergodic Schrödinger operators. Duke Math. J. 130(2) (2005) 393-400

[7] C. Baesens, J. Guckenheimer, S. Kim and R.S. MacKay, Three coupled oscillators: Mode-locking, global bifurcation and toroidal chaos. Physica D 49(3) (1991) 387-475

[8] D.G.M. Beersma, H.W. Broer, K.A. Cargar, K. Efstathiou and I. Hoveijn, Pacer cell response to periodic Zeitgebers. Preprint University of Groningen, 2011.

[9] M. Bennett, M.F. Schatz, H. Rockwood and K. Wiesenfeld, Huygens clocks. Proc. R. Soc. Lond. A 458 (2002) 563-579

[10] B.L.J. Braaksma and H.W. Broer, On a quasi-periodic Hopf bifurcation. Annales Insitut Henri Poincaré, Analyse non linéaire 4 (1987) 115-168 
[11] B.L.J. Braaksma, H.W. Broer and G.B. Huitema, Towards a quasi-periodic bifurcation theory. Mem. AMS 83(421) (1990), 81175

[12] H.W. Broer, KAM theory: the legacy of Kolmogorov's 1954 paper. Bull. AMS (New Series) 41(4) (2004) 507-521

[13] H.W. Broer, Normal forms in perturbation theory. In: R. Meyers (ed.), Encyclopadia of Complexity \& System Science. Springer (2009) 6310-6329

[14] H.W. Broer, M.C. Ciocci, H. Hanßmann and A. Vanderbauwhede, Quasiperiodic stability of normally resonant tori. Physica D (2009) 309318

[15] H.W. Broer, B. Hasselblatt and F. Takens (eds.): Handbook of Dynamical Systems Volume 3 North-Holland 2010.

[16] H.W. Broer, H.A. Dijkstra, C. Simó, A.E. Sterk and R. Vitolo, The dynamics of a low-order model for the Atlantic Multidecadal Oscillation. DCDS-B 16(1) (2011) 73102

[17] H.W. Broer, K. Efstathiou, and E. Subramanian, Robustness of unstable attractors in arbitrarily sized pulse-coupled systems with delay. Nonlinearity 21 (2008) 1349

[18] H.W. Broer, K. Efstathiou, and E. Subramanian, Heteroclinic cycles between unstable attractors. Nonlinearity 21 (2008) 13851410

[19] H.W. Broer, M. Golubitsky and G. Vegter, The geometry of resonance tongues: A Singularity Theory approach. Nonlinearity 16 (2003) 15111538

[20] H.W. Broer, M. Golubitsky, and G. Vegter. Geometry of resonance tongues. Singularity Theory. Proceedings of the 2005 Marseille Singularity School and Conference, pages 327-356, 2007.

[21] H.W. Broer, H. Hanßmann and J. Hoo, The quasi-periodic Hamiltonian Hopf bifurcation. Nonlinearity 20 (2007) 417-460

[22] H.W. Broer, H. Hanßmann, Á. Jorba, J. Villanueva and F.O.O. Wagener, Normal-internal resonances in quasi-periodically forces oscillators: a conservative approach. Nonlinearity 16 (2003) 1751-1791 
[23] H.W. Broer, H. Hanßmann and J. You, On the destruction of resonant Lagrangean tori in Hamiltonian systems. Preprint Universiteit Utrecht (2008)

[24] H.W. Broer, H. Hanßmann and F.O.O. Wagener, Quasi-periodic bifurcation theory: the geometry of KAM. 2012 (to appear)

[25] H.W. Broer, S.J. Holtman and G. Vegter, Recognition of the bifurcation type of resonance in a mildly degenerate Hopf-Neĭmark-Sacker families. Nonlinearity 21 (2008) 2463-2482

[26] H.W. Broer, S.J. Holtman, G. Vegter and R. Vitolo, Geometry and dynamics of mildly degenerate Hopf-Neĭmarck-Sacker families near resonance. Nonlinearity 22 (2009) 2161-2200

[27] H.W. Broer, S.J. Holtman and G. Vegter, Recognition of resonance type in periodically forced oscillators. Physica-D 239(17) (2010) 1627-1636

[28] H.W. Broer, S.J. Holtman, G. Vegter and R. Vitolo, Dynamics and Geometry Near Resonant Bifurcations. Regular and Chaotic Dynamics 16(1-2) (2011) 3950

[29] H.W. Broer, J. Hoo and V. Naudot, Normal linear stability of quasi-periodic tori. J. Differential Equations 232 (2007) 355-418

[30] H.W. Broer, G.B. Huitema and F. Takens, Unfoldings and bifurcations of quasi-periodic tori. Memoirs American Mathematical Society 83 \# 421 (1990) 1-82

[31] H.W. Broer and M. Levi, Geometrical aspects of stability theory for Hill's equations. Archive Rat. Mech. An. 131 (1995) 225-240

[32] H.W. Broer, J. Puig and C. Simó, Resonance tongues and instability pockets in the quasi-periodic Hill-Schrödinger equation. Commun. Math. Phys. 241 (2003) 467-503

[33] H.W. Broer and R. Roussarie, Exponential confinement of chaos in the bifurcations sets of real analytic diffeomorphisms. In: H.W. Broer, B. Krauskopf and G. Vegter (eds.) Global Analysis of Dynamical Systems. IoP Publishing (2001) 167-210 
[34] H.W. Broer and C. Simó, Hill's equation with quasi-periodic forcing: resonance tongues, instability pockets and global phenomena. Boletim Sociedade Brasileira Matemática 29 (1998) 253-293

[35] H.W. Broer and C. Simó, Resonance tongues in Hill's equations: a geometric approach. Journal of Differential Equations 166 (2000) 290-327

[36] H.W. Broer, C. Simó and J.C. Tatjer, Towards global models near homoclinic tangencies of dissipative diffeomorphisms. Nonlinearity 11(3) (1998) 667-770

[37] H.W. Broer, C. Simó and R. Vitolo, Bifurcations and strange attractors in the Lorenz-84 climate model with seasonal forcing. Nonlinearity 15(4) (2002) 1205-1267

[38] H.W. Broer, C. Simó and R. Vitolo, The Hopf-Saddle-Node bifurcation for fixed points of 3D-diffeomorphisms, analysis of a resonance 'bubble'. Physica D 237 (2008) 1773-1799

[39] H.W. Broer, C. Simó and R. Vitolo, The Hopf-Saddle-Node bifurcation for fixed points of 3D-diffeomorphisms, the Arnol'd resonance web. Bull. Belgian Math. Soc. Simon Stevin 15 (2008) 769-787

[40] H.W. Broer, C. Simó and R. Vitolo, Chaos and quasi-periodicity in diffeomorphisms of the solid torus. DCDS-B 14(3) (2010) 871-905

[41] H.W. Broer and F. Takens, Formally symmetric normal forms and genericity. Dynamics Reported 2 (1989) 36-60

[42] H.W. Broer and F. Takens, Dynamical Systems and Chaos. Appl. Math. Sc. 172 Springer 2011

[43] H.W. Broer and G. Vegter, Subordinate Sil'nikov bifurcations near some singularities of vector fields having low codimension. Ergodic Theory Dynamical Systems 4 (1984) 509-525

[44] H.W. Broer and G. Vegter, Bifurcational aspects of parametric resonance. Dynamics Reported, New Series 1 (1992) 1-51

[45] H.W. Broer and G. Vegter, Generic Hopf-Nel̆mark-Sacker bifurcations in feed forward systems. Nonlinearity 21 (2008) 1547-1578 
[46] A. Chenciner, Bifurcations de points fixes elliptiques. I. Courbes invariantes. Publ. Math. IHÉS 61 (1985) 67-127

[47] A. Chenciner, Bifurcations de points fixes elliptiques. II. Orbites périodiques et ensembles de Cantor invariants. Invent. Math. 80 (1985) 81106

[48] A. Chenciner, Bifurcations de points fixes elliptiques. III. Orbites périodiques de "petites" périodes et élimination résonnante des couples de courbes invariantes. Publ. Math. IHÉS 66 (1988) 5-91

[49] M.C. Ciocci, A. Litvak-Hinenzon and H.W. Broer, Survey on dissipative KAM theory including quasi-periodic bifurcation theory based on lectures by Henk Broer. In: J. Montaldi and T. Ratiu (Eds.): Geometric Mechanics and Symmetry: the Peyresq Lectures. LMS Lecture Notes Series, 306 Cambridge University Press (2005) 303-355

[50] A. Correia and J. Laskar, Mercury's capture into the 3/2 spin-orbit resonance as a result of its chaotic dynamics. Nature 429 (2004) 848-850

[51] R.H. Cushman and J.C. van der Meer, The Hamiltonian Hopf bifurcation in the Lagrange top. In: C. Albert (ed.) Géometrie Symplectique et Méchanique, Colloque International La Grande Motte, France, 23-28 Mai, 1988. Lecture Notes in Mathematics 1416 (1990) 26-38

[52] R.L. Devaney, An Introduction to Chaotic Dynamical Systems. 2nd Edition. Addison-Wesley 1989

[53] L.H. Eliasson, Floquet solutions for the one-dimensional quasi-periodic Schrödinger equation. Commun. Math. Phys. 146 (1992) 447-482

[54] M. Golubitsky and V. Guillemin, Stable Mappings and Their Singularities. Springer (1973)

[55] M. Golubitsky and D.G. Schaeffer, Singularities and Groups in Bifurcation Theory Vol. I. Springer (1985)

[56] M. Golubitsky, I. Stewart and D.G. Schaeffer, Singularities and Groups in Bifurcation Theory Vol. II. Appl. Math. Sc. 69 Springer (1988) 
[57] J. Guckenheimer and P. Holmes, Nonlinear Oscillations, Dynamical Systems, and Bifurcations of Vector Fields, Fifth Edition. Applied Mathematical Sciences 42, Springer 1997

[58] E. Hopf, A Mathematical Example Displaying Features of Turbulence. Comm. (Pure) Applied Mathematics 1 (1948) 303-322

[59] E. Hopf, Repeated branching through loss of stability, an example. In: J.B. Diaz (ed.) Proceedings of the conference on differential equations, Maryland 1955. University Maryland book store (1956) 49-56

[60] C. Huygens, Evvres complètes de Christiaan Huygens. (1888-1950) Vol. 5, 241-263 and Vol. 17, 156-189. Martinus Nijhoff 1888-1950

[61] Yu.A. Kuznetsov, Elements of Applied Bifurcation Theory, Third Edition. Applied Mathematical Sciences 112, Springer 2004

[62] L.D. Landau, On the problem of turbulence. Doklady Akademii Nauk SSSR 44 (1944) 339-342

[63] L.D. Landau and E.M. Lifshitz, Fluid Mechanics, Second Edition. Pergamon (1987)

[64] S.E. Newhouse, J. Palis and F. Takens, Bifurcations and stability of families of diffeomorphisms. Publ. Math. IHES 57 (1983) 5-71

[65] S.E. Newhouse, D. Ruelle and F. Takens, Occurrence of strange Axiom A attractors near quasi-periodic flows on $\mathbb{T}^{m}, m \leq 3$. Commun. Math. Phys. 64 (1978) 35-40

[66] B.B. Mandelbrot, The Fractal Geometry of Nature. Freeman (1977).

[67] J.K. Moser, On invariant curves of area-preserving mappings of an annulus. Nachrichten Akademie Wissenschaften Göttingen, Mathematisch-Physikalische Klasse II. 1 (1962) 1-20

[68] J.K. Moser, Lectures on Hamiltonian systems. Memoirs American Mathematical Society 81 (1968) 1-60

[69] J.K. Moser, Stable and random motions in dynamical systems, with special emphasis to celestial mechanics, Annals Mathematical Studies 77 (1973) Princeton University Press. 
[70] J.K. Moser and J. Pöschel, An extension of a result by Dinaburg and Sinai on quasi-periodic potentials. Comment. Math. Helvetici 59 (1984) 39-85

[71] Z. Nitecki, Differentiable dynamics. An introduction to the orbit structure of diffeomorphisms. Massachusetts Institute of Technology Press 1971.

[72] J. Oxtoby, Measure and Category. Springer 1971

[73] J. Palis, W.C. de Melo, Geometric Theory of Dynamical Systems. Springer 1982

[74] J. Palis and F. Takens, Hyperbolicity \& sensitive chaotic dynamics at homoclinic bifurcations. Cambridge University Press (1993)

[75] J.H. Poincaré, Les Méthodes Nouvelles de la Mécanique Céleste I, II, III. Gauthier-Villars (1892, 1893, 1899). Republished by Blanchard (1987).

[76] A. Pogromsky, D. Rijlaarsdam and H. Nijmeijer, Experimental Huygens synchronization of oscillators. In: M. Thiel, J. Kurths, M.C. Romano, A. Moura and G. Károlyi, Nonlinear Dynamics and Chaos: Advances and Perspectives. Springer Complexity (2010) 195-210

[77] B. van der Pol, De amplitude van vrije en gedwongen triode-trillingen. Tijdschrift Nederlands Radiogenooschap 1 (1920) 3-31

[78] B. van der Pol, The nonlinear theory of electric oscillations. Proceedings Institute Radio England 22 (1934) 1051-1086; Reprinted in: Selected Scientific Papers. North-Holland 1960

[79] D. Ruelle and F. Takens, On the nature of turbulence. Comm. Math. Phys. 20 (1971) 167-192; 23 (1971) 343-344

[80] J.A. Sanders, F. Verhulst and J. Murdock, Averaging Methods in Nonlinear Dynamical Systems, Revised Second Edition. Applied Mathematical Sciences 59, Springer (2007)

[81] C.L. Siegel and J.K. Moser, Lectures on Celestial Mechanics. Springer (1971)

[82] B. Simon, Operators with singular continuous spectrum: I. General operators. Ann. of Math. 141 (1995) 131-145 
[83] W. de Sitter, On the libration of the three inner large satellites of Jupiter. Publ. Astr. Lab. Groningen 17 (1907) 1-119

[84] W. de Sitter, New mathematical theory of Jupiters satellites. Ann. Sterrewacht Leiden XII (1925)

[85] A.E. Sterk, R. Vitolo, H.W. Broer, C. Simó and H.A. Dijkstra, New nonlinear mechanisms of midlatitude atmospheric low-frequency variability. Physica D 239(10) (2010) 702718

[86] J.J. Stoker, Nonlinear Vibrations in Mechanical and Electrical Systems. 2nd Ed. Wiley 1992

[87] S.H.Strogatz, Nonlinear Dynamics and Chaos. Addison Wesley 1994

[88] F. Takens, Forced oscillations and bifurcations. In: Applications of Global Analysis I, Comm. of the Math. Inst. Rijksuniversiteit Utrecht (1974). In: H.W. Broer, B. Krauskopf and G. Vegter (Eds.), Global Analysis of Dynamical Systems IoP Publishing (2001) 1-62

[89] R. Thom, Stabilité Structurelle et Morphogénèse. Benjamin 1972

[90] F. Verhulst, Nonlinear Differential Equations and Dynamical Systems. 2nd Edition. Universitext Springer 1996

[91] R. Vitolo, H.W. Broer, and C. Simó, Quasi-periodic bifurcations of invariant circles in low-dimensional dissipative dynamical systems. Regular and Chaotic Dynamics 16(1-2) (2011) 154184

[92] A. Vanderbauwhede. Branching of periodic solutions in time-reversible systems. In H.W. Broer and F. Takens, editors, Geometry and Analysis in Non-Linear Dynamics, volume 222 of Pitman Research Notes in Mathematics, pages 97-113. Pitman, London, 1992.

[93] A. Vanderbauwhede. Subharmonic bifurcation at multiple resonances. In Proceedings of the Mathematics Conference, pages 254-276, Singapore, 2000. World Scientific. 


\section{A Equivariant Singularity Theory}

\section{A.1 Lyapunov-Schmidt reduction}

Our method for finding resonance tongues — and tongue boundaries — proceeds as follows. Find the region in parameter space corresponding to points where the map $P$ has a $q$-periodic orbit; that is, solve the equation $P^{q}(x)=x$. Using a method due to Vanderbauwhede (see [92, 93]), we can solve for such orbits by Lyapunov-Schmidt reduction. More precisely, a $q$-periodic orbit consists of $q$ points $x_{1}, \ldots, x_{q}$ where

$$
P\left(x_{1}\right)=x_{2}, \ldots, P\left(x_{q-1}\right)=x_{q}, P\left(x_{q}\right)=x_{1} .
$$

Such periodic trajectories are just zeroes of the map

$$
\widehat{P}\left(x_{1}, \ldots, x_{q}\right)=\left(P\left(x_{1}\right)-x_{2}, \ldots, P\left(x_{q}\right)-x_{1}\right) .
$$

Note that $\widehat{P}(0)=0$, and that we can find all zeroes of $\widehat{P}$ near the resonance point by solving the equation $\widehat{P}(x)=0$ by Lyapunov-Schmidt reduction. Note also that the map $\widehat{P}$ has $\mathbb{Z}_{q}$ symmetry. More precisely, define

$$
\sigma\left(x_{1}, \ldots, x_{q}\right)=\left(x_{2}, \ldots, x_{q}, x_{1}\right)
$$

Then observe that

$$
\widehat{P} \sigma=\sigma \widehat{P} .
$$

At 0 , the Jacobian matrix of $\widehat{P}$ has the block form

$$
J=\left(\begin{array}{rrrrrrr}
A & -I & 0 & 0 & \cdots & 0 & 0 \\
0 & A & -I & 0 & \cdots & 0 & 0 \\
& & & \vdots & & & \\
0 & 0 & 0 & 0 & \cdots & A & -I \\
-I & 0 & 0 & 0 & \cdots & 0 & A
\end{array}\right)
$$

where $A=(d P)_{0}$. The matrix $J$ automatically commutes with the symmetry $\sigma$ and hence $J$ can be block diagonalized using the isotypic components of irreducible representations of $\mathbb{Z}_{q}$. (An isotypic component is the sum of the $\mathbb{Z}_{q}$ isomorphic representations. See [56] for details. In this instance all calculations 
can be done explicitly and in a straightforward manner.) Over the complex numbers it is possible to write these irreducible representations explicitly. Let $\omega$ be a $q^{t h}$ root of unity. Define $V_{\omega}$ to be the subspace consisting of vectors

$$
[x]_{\omega}=\left(\begin{array}{r}
x \\
\omega x \\
\vdots \\
\omega^{q-1} x
\end{array}\right) .
$$

A short calculation shows that

$$
J[x]_{\omega}=[(A-\omega I) x]_{\omega} .
$$

Thus $J$ has zero eigenvalues precisely when $A$ has $q^{\text {th }}$ roots of unity as eigenvalues. By assumption, $A$ has just one such pair of complex conjugate $q^{\text {th }}$ roots of unity as eigenvalues.

Since the kernel of $J$ is two-dimensional - by the simple eigenvalue assumption in the Hopf bifurcation - it follows using Lyapunov-Schmidt reduction that solving the equation $\widehat{P}(x)=0$ near a resonance point is equivalent to finding the zeros of a reduced map from $\mathbb{R}^{2} \rightarrow \mathbb{R}^{2}$. We can, however, naturally identify $\mathbb{R}^{2}$ with $\mathbb{C}$, which we do. Thus we need to find the zeros of a smooth implicitly defined function

$$
g: \mathbb{C} \rightarrow \mathbb{C},
$$

where $g(0)=0$ and $(d g)_{0}=0$. Moreover, assuming that the Lyapunov-Schmidt reduction is done to respect symmetry, the reduced map $g$ commutes with the action of $\sigma$ on the critical eigenspace. More precisely, let $\omega$ be the critical resonant eigenvalue of $(d P)_{0}$; then

$$
g(\omega z)=\omega g(z)
$$

Since $p$ and $q$ are coprime, $\omega$ generates the group $\mathbb{Z}_{q}$ consisting of all $q^{\text {th }}$ roots of unity. So $g$ is $\mathbb{Z}_{q}$-equivariant.

We propose to use $\mathbb{Z}_{q}$-equivariant singularity theory to classify resonance tongues and tongue boundaries.

\section{A.2 Equivariant Singularity Theory}

In this section we develop normal forms for the simplest singularities of $\mathbb{Z}_{q^{-}}$ equivariant maps $g$ of the form (18), as presented in Section 3.1. To do this, we need to describe the form of $\mathbb{Z}_{q}$-equivariant maps, contact equivalence, and finally the normal forms. 
The structure of $\mathbb{Z}_{q}$-equivariant maps. We begin by determining a unique form for the general $\mathbb{Z}_{q}$-equivariant polynomial mapping. By Schwarz's theorem [56] this representation is also valid for $C^{\infty}$ germs.

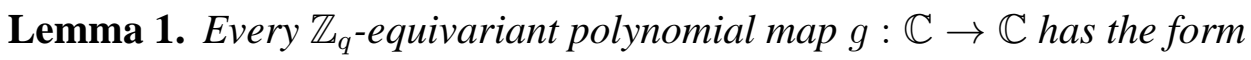

$$
g(z)=K(u, v) z+L(u, v) \bar{z}^{q-1},
$$

where $u=z \bar{z}, v=z^{q}+\bar{z}^{q}$, and $K, L$ are uniquely defined complex-valued function germs.

$\mathbb{Z}_{q}$ contact equivalences. Singularity theory approaches the study of zeros of a mapping near a singularity by implementing coordinate changes that transform the mapping to a 'simple' normal form and then solving the normal form equation. The kinds of transformations that preserve the zeros of a mapping are called contact equivalences. More precisely, two $\mathbb{Z}_{q^{-}}$equivariant germs $g$ and $h$ are $\mathbb{Z}_{q^{-}}$ contact equivalent if

$$
h(z)=S(z) g(Z(z))
$$

where $Z(z)$ is a $\mathbb{Z}_{q}$-equivariant change of coordinates and $S(z): \mathbb{C} \rightarrow \mathbb{C}$ is a real linear map for each $z$ that satisfies

$$
S(\gamma z) \gamma=\gamma S(z)
$$

for all $\gamma \in \mathbb{Z}_{q}$.

Normal form theorems. In this section we consider two classes of normal forms - the codimension two standard for resonant Hopf bifurcation and one more degenerate singularity that has a degeneracy at cubic order. These singularities all satisfy the nondegeneracy condition $L(0,0) \neq 0$; we explore this case first.

Theorem 2. Suppose that

$$
h(z)=K(u, v) z+L(u, v) \bar{z}^{q-1}
$$

where $K(0,0)=0$.

1. Let $q \geq 5$. If $K_{u} L(0,0) \neq 0$, then $h$ is $\mathbb{Z}_{q}$ contact equivalent to

$$
g(z)=|z|^{2} z+\bar{z}^{q-1}
$$

with universal unfolding

$$
G(z, \sigma)=\left(\sigma+|z|^{2}\right) z+\bar{z}^{q-1} .
$$


2. Let $q \geq 7$. If $K_{u}(0,0)=0$ and $K_{u u}(0,0) L(0,0) \neq 0$, then $h$ is $\mathbb{Z}_{q}$ contact equivalent to

$$
g(z)=|z|^{4} z+\bar{z}^{q-1}
$$

with universal unfolding

$$
G(z, \sigma, \tau)=\left(\sigma+\tau|z|^{2}+|z|^{4}\right) z+\bar{z}^{q-1},
$$

where $\sigma, \tau \in \mathbb{C}$.

Remark. Normal forms for the cases $q=3$ and $q=4$ are slightly different. See [19] for details.

\section{A.3 Resonances in forced oscillators}

As indicated in Section 2.1 subharmonic bifurcations in periodicially forced oscillators and the can be studied by lifting the system to a suitable covering space. This section contains the details of this procedure.

Hopf-Nermark-Sacker bifurcations in forced oscillators. Forced oscillators depending on parameters may undergo bifurcations involving the birth or death of subharmonics. In particular, we consider $2 \pi$-periodic systems on $\mathbb{C}$ of the form

$$
\dot{z}=F(z, \bar{z}, \mu)+\varepsilon G(z, \bar{z}, t, \mu),
$$

obtained from an autonomous system by a small $2 \pi$-periodic perturbation. Here $\varepsilon$ is a real perturbation parameter, and $\mu \in \mathbb{R}^{k}$ is an additional $k$-dimensional parameter. Subharmonics of order $q$ may appear or disappear upon variation of the parameters if the linear part of $F$ at $z=0$ satisfies a $p: q$-resonance condition which is appropriately detuned upon variation of the parameter $\mu$.

The Hopf-Nel̆mark-Sacker Normal form of such systems reveals this type of bifurcation. To this end, consider a $2 \pi$-periodic forced oscillator on $\mathbb{C}$ of the form

$$
\dot{z}=X(z, \bar{z}, t, \mu),
$$

where

$$
X(z, \bar{z}, t, \mu)=i \omega_{N} z+(\alpha+i \delta) z+z P(z, \bar{z}, \mu)+\varepsilon Q(z, \bar{z}, t, \mu) .
$$

Here $\mu \in \mathbb{R}^{k}$, and $\varepsilon$ is a small real parameter. Furthermore we assume that $P$ and $Q$ contain no terms that are independent of $z$ and $\bar{z}$ (i.e., $P(0,0, \mu)=0$ and 
$Q(0,0, t, \mu)=0)$, and that $Q$ does not even contain terms that are linear in $z$ and $\bar{z}$. Any system of the form (19) with linear part $\dot{z}=i \omega_{N} z$ can be brought into this form after a straightforward initial transformation. See [30, Part II] for details. Applying the algorithm of, e.g., [20], this system can be brought into the following Hopf-Neĭmark-Sacker normal form:

\section{Theorem 3. (Normal Form to order $q$ )}

The system (20) has normal form

$$
\dot{z}=i \omega_{N} z+(\alpha+i \delta) z+z F\left(|z|^{2}, \mu\right)+d \varepsilon \bar{z}^{q-1} e^{i p t}+O(q+1),
$$

where $F\left(|z|^{2}, \mu\right)$ is a complex polynomial of degree $q-1$ with $F(0, \mu)=0$, and $d$ is a complex constant.

Subharmonics of order $q$ are to be expected if the linear part satisfies a $p: q$ -

resonance condition, in other words, if the normal frequency $\omega_{N}$ is equal to $\frac{p}{q}$ (with $p$ and $q$ relatively prime). To explore these subharmonics further we apply the Van der Pol transformation and bring the Poincaré-map of the lifted system into its Takens Normal Form.

Existence of $2 \pi q$-periodic orbits. The Van der Pol transformation. Subharmonics of order $q$ of the $2 \pi$-periodic forced oscillator (20) correspond to $q$ periodic orbits of the Poincaré time $2 \pi$-map $P: \mathbb{C} \rightarrow \mathbb{C}$. These periodic orbits of the Poincaré map are brought into one-one correspondence with the zeros of a vector field on a $q$-sheeted cover of the phase space $\mathbb{C} \times \mathbb{R} /(2 \pi \mathbb{Z})$ via the Van der Pol transformation, cf [44]. This transformation corresponds to a $q$-sheeted covering

$$
\begin{aligned}
\Pi: \mathbb{C} \times \mathbb{R} /(2 \pi q \mathbb{Z}) & \rightarrow \mathbb{C} \times \mathbb{R} /(2 \pi \mathbb{Z}), \\
(z, t) & \mapsto\left(z \mathrm{e}^{i t p / q}, t(\bmod 2 \pi \mathbb{Z})\right)
\end{aligned}
$$

with cyclic Deck group of order $q$ generated by

$$
(z, t) \mapsto\left(z \mathrm{e}^{2 \pi i p / q}, t-2 \pi\right) .
$$

The Van der Pol transformation $\zeta=z e^{-i \omega_{N} t}$ lifts the forced oscillator (19) to the system

$$
\dot{\zeta}=(\alpha+i \delta) \zeta+\zeta P\left(\zeta e^{i \omega_{N} t}, \bar{\zeta} e^{-i \omega_{N} t}, \mu\right)+\varepsilon Q\left(\zeta e^{i \omega_{N} t}, \bar{\zeta} e^{-i \omega_{N} t}, t, \mu\right)
$$


on the covering space $\mathbb{C} \times \mathbb{R} /(2 \pi q \mathbb{Z})$. The latter system is $\mathbb{Z}_{q}$-equivariant. A straightforward application of (23) to the normal form (21) yields the following normal form for the lifted forced oscillator.

Theorem 4. (Equivariant Normal Form of order $q$ )

On the covering space, the lifted forced oscillator has the $\mathbb{Z}_{q}$-equivariant normal form:

$$
\dot{\zeta}=(\alpha+i \delta) \zeta+\zeta F\left(|\zeta|^{2}, \mu\right)+d \varepsilon \bar{\zeta}^{q-1}+O(q+1),
$$

where the $O(q+1)$ terms are $2 \pi q$-periodic.

Resonance tongues for families of forced oscillators. Bifurcations of $q$-periodic orbits of the Poincaré map $P$ on the base space correspond to bifurcations of fixed points of the Poincaré map $\tilde{P}$ on the $q$-sheeted covering space introduced in connection with the Van der Pol transformation (22). Denoting the normal form system (21) on the base space by $\mathcal{N}$, and the normal form system (24) of the lifted forced oscillator by $\tilde{\mathcal{N}}$, we see that

$$
\Pi_{*} \tilde{\mathcal{N}}=\mathcal{N} .
$$

The Poincaré mapping $\tilde{P}$ of the normal form on the covering space now is the $2 \pi q$-period mapping

$$
\tilde{P}=\tilde{\mathcal{N}}^{2 \pi q}+O(q+1),
$$

where $\tilde{\mathcal{N}}^{2 \pi q}$ denotes the $2 \pi q$-map of the (planar) vector field $\tilde{\mathcal{N}}$. Following the Corollary to the Normal Form Theorem of [44, page 12], we conclude for the original Poincaré map $P$ of the vector field $X$ on the base space that

$$
P=R_{2 \pi \omega_{N}} \circ \tilde{\mathcal{N}}^{2 \pi}+O(q+1),
$$

where $R_{2 \pi \omega_{N}}$ is the rotation over $2 \pi \omega_{N}=2 \pi p / q$, which precisely is the Takens Normal Form of $P$ at $(z, \mu)=(0,0)$, see [88].

Our interest is with the $q$-periodic points of $P_{\mu}$, which correspond to the fixed points of $\tilde{P}_{\mu}$. This fixed point set and the boundary thereof in the parameter space $\mathbb{R}^{3}=\{a, \delta, \varepsilon\}$ is approximately described by the discriminant set of

$$
(a+i \delta) \zeta+\zeta \tilde{F}\left(|\zeta|^{2}, \mu\right)+\varepsilon d \bar{\zeta}^{q-1},
$$

which is the truncated right hand side of (24). This gives rise to the bifurcation equation that determine the boundaries of the resonance tongues. The following theorem implies that, under the conditions that $d \neq 0 \neq F_{u}(0,0)$, the order of tangency at the tongue tips is generic. Here $F_{u}(0,0)$ is the partial derivative of $F(u, \mu)$ with respect to $u$. 
Theorem 5. (Bifurcation equations modulo contact equivalence)

Assume that $d \neq 0$ and $F_{u}(0,0) \neq 0$. Then the polynomial (24) is $\mathbb{Z}_{q}$-equivariantly contact equivalent with the polynomial

$$
G(\zeta, \mu)=\left(a+i \delta+|\zeta|^{2}\right) \zeta+\varepsilon \bar{\zeta}^{q-1} .
$$

The discriminant set of the polynomial $G(\zeta, \mu)$ is of the form

$$
\delta= \pm \varepsilon(-a)^{(q-2) / 2}+O\left(\varepsilon^{2}\right) .
$$

Proof. The polynomial (25) is a universal unfolding of the germ $|\zeta|^{2} \zeta+\varepsilon \bar{\zeta}^{q-1}$ under $\mathbb{Z}_{q}$ contact equivalence. See [19] for a detailed computation. The tongue boundaries of a $p: q$ resonance are given by the bifurcation equations

$$
\begin{aligned}
G(\zeta, \mu) & =0, \\
\operatorname{det}(d G)(\zeta, \mu) & =0 .
\end{aligned}
$$

As in [19, Theorem 3.1] we put $u=|z|^{2}$, and $b(u, \mu)=a+i \delta+u$. Then $G(\zeta, \mu)=b(u, \mu) \zeta+\varepsilon \bar{\zeta}^{q-1}$. According to (the proof of) [19, Theorem 3.1], the system of bifurcation equations is equivalent to

$$
\begin{aligned}
|b|^{2} & =\varepsilon^{2} u^{q-2}, \\
b \bar{b}^{\prime}+\bar{b} b^{\prime} & =(q-2) \varepsilon^{2} u^{q-3},
\end{aligned}
$$

where $b^{\prime}=\frac{\partial b}{\partial u}(u, \mu)$. A short computation reduces the latter system to the equivalent

$$
\begin{aligned}
(a+u)^{2}+\delta^{2} & =\varepsilon^{2} u^{q-2} \\
a+u & =\frac{1}{2}(q-2) \varepsilon^{2} u^{q-3} .
\end{aligned}
$$

Eliminating $u$ from this system of equations yields expression (26) for the tongue boundaries.

The discriminant set of the equivariant polynomial (25) forms the boundary of the resonance tongues. See Figure 15. At this surface we expect the HopfNermark-Sacker bifurcation to occur; here the Floquet exponents of the linear part of the forced oscillator cross the complex unit circle. This bifurcation gives rise to an invariant 2-torus in the $3 \mathrm{D}$ phase space $\mathbb{C} \times \mathbb{R} /(2 \pi \mathbb{Z})$. Resonances occur when the eigenvalues cross the unit circle at roots of unity $\mathrm{e}^{2 \pi i p / q}$. 'Inside' the tongue the 2-torus is phase-locked to subharmonic periodic solutions of order $q$. 

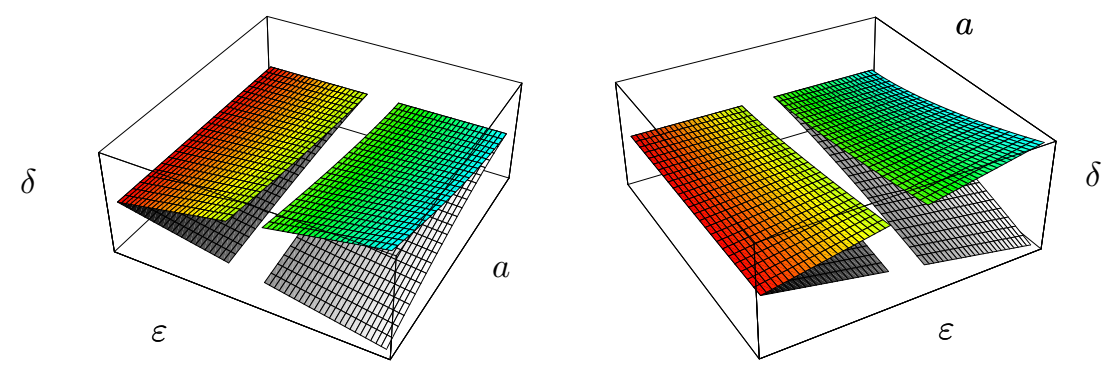

Figure 15: Resonance zones for forced oscillator families: the Hopf-NeĭmarkSacker phenomenon. 